\title{
SCATTERING FOR NONLINEAR SCHRÖDINGER EQUATION UNDER PARTIAL HARMONIC CONFINEMENT
}

\author{
PAOLO ANTONELLI, RÉMI CARLES, AND JORGE DRUMOND SILVA
}

\begin{abstract}
We consider the nonlinear Schrödinger equation under a partial quadratic confinement. We show that the global dispersion corresponding to the direction(s) with no potential is enough to prove global in time Strichartz estimates, from which we infer the existence of wave operators thanks to suitable vector-fields. Conversely, given an initial Cauchy datum, the solution is global in time and asymptotically free, provided that confinement affects one spatial direction only. This stems from anisotropic Morawetz estimates, involving a marginal of the position density.
\end{abstract}

\section{INTRODUCTION}

1.1. Motivation. It is well known that the solution to

$$
i \partial_{t} u+\frac{1}{2} \Delta u=0, \quad(t, x) \in \mathbf{R} \times \mathbf{R}^{d},
$$

with $u_{\mid t=0} \in \mathcal{S}\left(\mathbf{R}^{d}\right)$, has large time dispersive properties which allow, for instance, to develop scattering theories both in the presence of a linear perturbation and in a nonlinear setting (see e.g. [9, 11, 14, 33]). On the other hand, in the presence of an (isotropic) harmonic potential,

$$
i \partial_{t} u+\frac{1}{2} \Delta u=\frac{|x|^{2}}{2} u, \quad(t, x) \in \mathbf{R} \times \mathbf{R}^{d},
$$

the solution is periodic in time. This is a consequence of the existence of a Hilbert basis for the harmonic oscillator $H_{0}=-\frac{1}{2} \Delta+\frac{|x|^{2}}{2}$ (see e.g. [23]): the pure point spectrum is

$$
\sigma_{p}\left(H_{0}\right)=\left\{\frac{d}{2}+k=: \lambda_{k} ; k \in \mathbf{N}\right\},
$$

and the associated eigenfunctions are given by (tensor products of) Hermite functions (denoted by $\psi_{j k}$, associated to $\lambda_{k}$ ), which form a basis of $L^{2}\left(\mathbf{R}^{d}\right)$. So if

$$
u(0, x)=\sum_{j, k \in \mathbf{N}} \alpha_{j k} \psi_{j k}(x),
$$

then for all $t \in \mathbf{R}$,

$$
u(t, x)=\sum_{j, k \in \mathbf{N}} \alpha_{j k} \psi_{j k}(x) e^{-i\left(\frac{d}{2}+k\right) t}
$$

2000 Mathematics Subject Classification. Primary: 35Q55. Secondary: 35B40, 35P25, 81U30.

This work was supported by the French ANR project SchEq (ANR-12-JS01-0005-01). J. Drumond Silva was partially supported by the Center for Mathematical Analysis, Geometry and Dynamical Systems-LARSys through the Fundação para a Ciência e Tecnologia (FCT/Portugal) Program POCTI/FEDER. 
Perturbations of 1.2 rather lead to KAM type results, in the linear or in the nonlinear setting (see e.g. [17, 22]). In this paper, we study the large time behavior of solutions to a nonlinear perturbation of a mixture of the above two linear dynamics:

$$
i \partial_{t} u=H u+\lambda|u|^{2 \sigma} u, \quad(x, y) \in \mathbf{R}^{n} \times \mathbf{R}^{d-n}, \quad H=-\frac{1}{2} \Delta_{x}+\frac{|x|^{2}}{2}-\frac{1}{2} \Delta_{y}
$$

with $d \geqslant 2,1 \leqslant n \leqslant d-1, \lambda \in \mathbf{C}$ and $\sigma>0$. We show that if the power of the nonlinearity is sufficiently large, then a scattering theory is available: if $f(z)=|z|^{2 \sigma} z$ is short range in space dimension $d-n$, then the nonlinearity is negligible for large time. In the rest of the introduction, we make this statement more precise.

We emphasize also the fact that the harmonic potential in (1.3) corresponds to the standard modelling for magnetic traps in the contexts of Bose-Einstein condensation (see e.g. [19, 28]). So for instance, our result shows that turning off the confinement in some of the directions may suffice for the condensate to evolve asymptotically freely, and that turning it off in all directions is not necessary: it is possible to keep some properties of the linear confinement, associated to the linear Hamiltonian $H$.

\subsection{A quick review on scattering for NLS. Consider}

$$
i \partial_{t} u+\frac{1}{2} \Delta u=\lambda|u|^{2 \sigma} u, \quad x \in \mathbf{R}^{d}
$$

and introduce the space

$$
\Sigma=\left\{f \in L^{2}\left(\mathbf{R}^{d}\right) ;\|f\|_{\Sigma}:=\|x f\|_{L^{2}\left(\mathbf{R}^{d}\right)}+\|\nabla f\|_{L^{2}\left(\mathbf{R}^{d}\right)}<\infty\right\} .
$$

The following statement summarizes several results which can be found in e.g. [9, 14].

Theorem 1.1. Let $d \geqslant 1, \lambda \in \mathbf{C}, \sigma>0$ with $\sigma<2 /(d-2)$ if $d \geqslant 3$.

1. Existence of wave operators in $H^{1}$. Suppose that $\sigma \geqslant 2 / d$. Let $u_{-} \in H^{1}\left(\mathbf{R}^{d}\right)$. There exist $T>0$, depending on $|\lambda|, d, \sigma$ and $\left\|u_{-}\right\|_{H^{1}}$, and a unique solution $u \in$ $C\left((-\infty,-T] ; H^{1}\right)$ to 1.4 such that

$$
\left\|u(t)-e^{i \frac{t}{2} \Delta} u_{-}\right\|_{H^{1}}=\left\|e^{-i \frac{t}{2} \Delta} u(t)-u_{-}\right\|_{H^{1}} \underset{t \rightarrow-\infty}{\longrightarrow} 0 .
$$

2. Existence of wave operators in $\Sigma$. Let $u_{-} \in \Sigma$. There exist $T>0$, depending on $|\lambda|, d$, $\sigma$ and $\left\|u_{-}\right\|_{\Sigma}$, and a unique solution $u \in C((-\infty,-T] ; \Sigma)$ to (1.4) such that

$$
\left\|e^{-i \frac{t}{2} \Delta} u(t)-u_{-}\right\|_{\Sigma} \underset{t \rightarrow \infty}{\longrightarrow} 0
$$

under the following assumption on $\sigma$ :

$$
\sigma>1 \text { if } d=1, \quad \sigma>\frac{2}{d+2} \text { if } d \geqslant 2 .
$$

3. Asymptotic completeness in $H^{1}$. Suppose that $\lambda \in \mathbf{R}$, with $\lambda \geqslant 0$, and $\sigma>2 / d$. For all $u_{0} \in H^{1}\left(\mathbf{R}^{d}\right)$, there exist a unique $u \in C\left(\mathbf{R} ; H^{1}\right)$ solution to (1.4) with $u_{\mid t=0}=u_{0}$, and a unique $u_{+} \in H^{1}\left(\mathbf{R}^{d}\right)$ such that

$$
\left\|u(t)-e^{i \frac{t}{2} \Delta} u_{+}\right\|_{H^{1}}=\left\|e^{-i \frac{t}{2} \Delta} u(t)-u_{+}\right\|_{H^{1}} \underset{t \rightarrow+\infty}{\longrightarrow} 0 .
$$

4. Asymptotic completeness in $\Sigma$. Suppose that $\lambda \geqslant 0$, and $\sigma \geqslant \frac{2-d+\sqrt{d^{2}+12 d+4}}{4 d}$. For all $u_{0} \in \Sigma$, there exist a unique $u \in C(\mathbf{R} ; \Sigma)$ solution to (1.4) with $u_{\mid t=0}=u_{0}$, and a unique $u_{+} \in \Sigma$ such that

$$
\left\|e^{-i \frac{t}{2} \Delta} u(t)-u_{+}\right\|_{\Sigma t \rightarrow+\infty} 0 .
$$


5. Asymptotic completeness in $\Sigma$ for small data. Let $\lambda \in \mathbf{R}$. Suppose that

$$
\sigma>1 \text { if } d=1, \quad \sigma>\frac{2}{d+2} \text { if } d \geqslant 2,
$$

and $\lambda \geqslant 0$ if $\sigma \geqslant 2 / d$. If $\left\|u_{0}\right\|_{\Sigma}$ is sufficiently small, there exist a unique $u \in C(\mathbf{R} ; \Sigma)$ solution to (1.4) with $u_{\mid t=0}=u_{0}$, and a unique $u_{+} \in \Sigma$ such that

$$
\left\|e^{-i \frac{t}{2} \Delta} u(t)-u_{+}\right\|_{\Sigma} \underset{t \rightarrow+\infty}{\longrightarrow} 0 .
$$

6. Existence of long range effects. Suppose $\sigma \leqslant 1 / d$. If $u \in C\left([T, \infty) ; L^{2}\left(\mathbf{R}^{d}\right)\right.$ solution to (1.4) and $u_{+} \in L^{2}\left(\mathbf{R}^{d}\right)$ satisfy

$$
\left\|u(t)-e^{i \frac{t}{2} \Delta} u_{+}\right\|_{L^{2}}=\left\|e^{-i \frac{t}{2} \Delta} u(t)-u_{+}\right\|_{L^{2}} \underset{t \rightarrow+\infty}{\longrightarrow} 0,
$$

then necessarily, $u=u_{+}=0$.

Remark 1.2. Uniqueness is actually granted in smaller spaces than in the above statement, involving a mixed time-space norm which we have omitted to simplify the presentation.

Remark 1.3. As pointed out in the above statement, the free group $e^{i \frac{t}{2} \Delta}$ is unitary on $H^{1}\left(\mathbf{R}^{d}\right)$, while it is not on $\Sigma$.

The above (positive) results all rely on (global in time) Strichartz estimates. It is essentially the only tool to prove the first point, while the second point uses the operator $x+i t \nabla$, which provides an explicit decay rate in time, to improve the assumption on $\sigma$. The third point relies on Morawetz estimates (see also [16, 29]). The fourth point relaxes the assumption of the third point on $\sigma$, since

$$
\frac{2}{d}>\frac{2-d+\sqrt{d^{2}+12 d+4}}{4 d}>\frac{1}{d}
$$

It relies on the pseudo-conformal evolution law, which provides a rather simple relation concerning the time evolution of the $L^{2}$ norm of $(x+i t \nabla) u$. The fifth point is essentially the same as the second one, plus a bootstrap argument. The final point means that if $\sigma \leqslant 1 / d$, linear and nonlinear dynamics can no longer be (easily) compared, due to long range effects.

To be complete, we recall the above mentioned pseudo-conformal evolution law: if $u$ solves (1.4), then

$$
\frac{d}{d t}\left(\frac{1}{2}\left\|\left(x+i t \nabla_{x}\right) u\right\|_{L^{2}}^{2}+\frac{\lambda t^{2}}{\sigma+1}\|u\|_{L^{2 \sigma+2}}^{2 \sigma+2}\right)=\frac{\lambda t}{\sigma+1}(2-d \sigma)\|u\|_{L^{2 \sigma+2}}^{2 \sigma+2} .
$$

This law was discovered in [15]. A generalization to the case of an external potential can be found in [9]. Note however that in the presence of an external potential, it seems that the corresponding evolution law can be exploited only in the case where the potential is exactly quadratic and isotropic ([5]). In particular, in the case of a partial confinement as in (1.3), the law presented in [9] seems helpless as far as the description of large time behavior is concerned.

1.3. Heuristics. Leaving out all the technical aspects, scattering for (1.4) stems from the following remark. As $t \rightarrow+\infty$, we have

$$
e^{i \frac{t}{2} \Delta} f(x)=\frac{1}{(i t)^{d / 2}} \widehat{f}\left(\frac{x}{t}\right) e^{i \frac{|x|^{2}}{2 t}}+o(1) \text { in } L^{2}\left(\mathbf{R}^{d}\right),
$$


where the Fourier transform is normalized as

$$
\mathcal{F} f(\xi)=\widehat{f}(\xi)=\frac{1}{(2 \pi)^{d / 2}} \int_{\mathbf{R}^{d}} f(x) e^{-i x \cdot \xi} d x .
$$

Assuming that the solution to (1.4) behaves as the free evolution of a scattering state $u_{+}$as $t \rightarrow+\infty$, the nonlinear potential in 1.4 satisfies

$$
|u(t, x)|^{2 \sigma} \approx \frac{1}{t^{d \sigma}}\left|\widehat{u}_{+}\left(\frac{x}{t}\right)\right|^{2 \sigma} .
$$

The function on the right hand side belongs to $L_{t}^{1} L_{x}^{\infty}$ provided that ( $u_{+}$is sufficiently localized and) $\sigma>1 / d$. From the linear scattering point of view [11], this is consistent with the fact that $u$ behaves asymptotically like a solution to the free equation (1.1). In the case $\sigma \leqslant 1 / d$, the (nonlinear) potential remains relevant at leading order for all time, no matter how large, as proved in [2]; long range effects were described for the first time in [27] in the case of 1.4. Note however that except in the cases $d=1$ and $d=2$, Theorem 1.1 introduces restrictions on the lower bound for $\sigma$ which are not intuitive, and are likely to be "only" technical.

In the case of (1.3), the $x$-part is not expected to yield large time dispersion, while the $y$ part should provide large time dispersion. From this perspective, it seems natural to expect a scattering theory for 1.3 with the same numerology as in Theorem 1.1 , with $d$ replaced by $d-n$ as far as lower bounds on $\sigma$ are concerned.

Such a problem is to be compared with the approach in [31]. The authors study [1.3] where, instead of considering the harmonic oscillator on $\mathbf{R}^{n}$, they examine the Laplacian on a compact manifold without boundary. Also, in [18], Z. Hani and B. Pausader consider a similar problem in the particular case where $\lambda=1, \sigma=2$ (quintic, defocusing nonlinearity), $x \in \mathbf{T}^{2}(n=2)$ and $d=3$. This problem is in addition both $L^{2}$ and energycritical, an aspect which introduces new difficulties compared to 1.3 as it is treated in the present paper. In both [18] and [31], the nonlinearity in supposed to be short range in the $y$ variable so that scattering in $H^{1}$ can be expected, $\sigma \geqslant 2 /(d-n)$, and small data scattering is established: for small initial data (in spaces that we do not describe here), the solution to the nonlinear equation is global in time, and behaves for large time like a solution to the linear equation.

More recently, in [32], the authors have considered the issue of asymptotic completeness for the nonlinear Schrödinger equation posed on $\mathbf{R}^{N} \times \mathbf{T}$, a case which corresponds to $n=1$ for 1.3 . They prove that a scattering theory is available with the same numerology as on $\mathbf{R}^{N}$, provided that the nonlinearity in energy-subcritical in total space dimension $N+1$. Such a result is qualitatively very similar to our Theorem 1.10

Note that like with $H$ in 1.3 , the dynamics in the $x$ variable prevents the free dynamics from being as trivial as in the case of 1.1. We will return to a comparison with these papers on a more technical level in Sections 2 and 3 .

1.4. Main results. Note that the definition of $\Sigma$ has to be adapted to the present notations:

$$
\Sigma=\left\{f \in L^{2}\left(\mathbf{R}^{d}\right) ;\|f\|_{\Sigma}:=\|x f\|_{L^{2}\left(\mathbf{R}^{d}\right)}+\|y f\|_{L^{2}\left(\mathbf{R}^{d}\right)}+\left\|\nabla_{x, y} f\right\|_{L^{2}\left(\mathbf{R}^{d}\right)}<\infty\right\} .
$$

In view of [7, Theorem 1.8], we have:

Proposition 1.4. Let $d \geqslant 2,1 \leqslant n \leqslant d-1, \lambda \in \mathbf{R}$, and $\sigma>0$ with $\sigma<2 /(d-2)$ if $d \geqslant 3$. If $\sigma \geqslant 2 / d$, assume in addition that $\lambda \geqslant 0$. For all $u_{0} \in \Sigma$, 1.3) has a unique solution

$$
u \in C(\mathbf{R} ; \Sigma) \cap L_{\mathrm{loc}}^{(4 \sigma+4) /(d \sigma)}\left(\mathbf{R} ; W^{1,2 \sigma+2}\left(\mathbf{R}^{d}\right)\right)
$$


such that $u_{\mid t=0}=u_{0}$. The following conservations hold:

$$
\frac{d}{d t}\|u(t)\|_{L^{2}\left(\mathbf{R}^{d}\right)}^{2}=\frac{d}{d t}\left(\langle u(t), H u(t)\rangle+\frac{\lambda}{\sigma+1}\|u(t)\|_{L^{2 \sigma+2}\left(\mathbf{R}^{d}\right)}^{2 \sigma+2}\right)=0 .
$$

Our first result regarding scattering theory is the existence of wave operators, along with asymptotic completeness for small data:

Theorem 1.5. Let $d \geqslant 2,1 \leqslant n \leqslant d-1, \lambda \in \mathbf{C}$, and $\sigma>0$ with $\sigma<2 /(d-2)$ if $d \geqslant 3$. Suppose in addition that

$$
\sigma>\frac{2 d}{d+2} \frac{1}{d-n}
$$

- Existence of wave operators. Let $u_{-} \in \Sigma$. There exist $T>0$, depending on $|\lambda|, d, n, \sigma$ and $\left\|u_{-}\right\|_{\Sigma}$, and a unique solution $u \in C((-\infty,-T] ; \Sigma)$ to $(1.3)$ such that

$$
\left\|e^{i t H} u(t)-u_{-}\right\|_{\Sigma} \underset{t \rightarrow \infty}{\longrightarrow} 0 \text {. }
$$

- Asymptotic completeness for small data. Let $u_{0} \in \Sigma$ and $u$ be the solution provided by Proposition 1.4 If $\left\|u_{0}\right\|_{\Sigma}$ is sufficiently small, there exists a unique $u_{+} \in \Sigma$ such that

$$
\left\|e^{i t H} u(t)-u_{+}\right\|_{\Sigma} \underset{t \rightarrow+\infty}{\longrightarrow} 0 .
$$

Remark 1.6 (Uniqueness). Uniqueness is stated rather loosely in the first point, for an extra property will be needed, which is too involved to be made precise here. See Section 4 for full details.

Remark 1.7 (Lower bound on $\sigma$ ). The assumption 1.5 is the expected one, except possibly for the factor $2 d /(d+2)$. If $d=2$ (hence $n=1)$, 1.5 corresponds to the heuristics in the previous subsection. On the other hand if $d \geqslant 3$, we have $2 d /(d+2)>1$, and the expected lower bound for $\sigma$ is not reached. Note that this factor is the same as in the second point in Theorem 1.1. It actually appears for the same technical reason as in the context of Theorem 1.1 as will be clear in Section 4

Remark 1.8 (Values allowed for $n$ ). The assumptions of Theorem 1.5 always allow the value $n=1$ (one direction of confinement). The value $n=2$ is allowed for $d \geqslant 3$, since the range

$$
\frac{2 d}{d+2} \frac{1}{d-2}<\sigma<\frac{2}{d-2}
$$

is non-empty. The value $n=3$ is allowed only if $d \geqslant 7$, and no value $n \geqslant 4$ can be considered, since for $d \geqslant 5$,

$$
\frac{2 d}{d+2} \frac{1}{d-4}>\frac{2}{d-2}
$$

So in the physically relevant cases $d=2$ or 3 , any $n$ such that $1 \leqslant n \leqslant d-1$ is allowed.

Regarding asymptotic completeness for large data, the first remark is that due to the anisotropy of the operator $H$, no analogue of the pseudo-conformal evolution law, which is used in the proof of the fourth point in Theorem 1.1, must be expected. Therefore, it is more sensible to rely on Morawetz type estimates, which we prove in a more general context than the framework of 1.3 with a quadratic potential.

Proposition 1.9 (Anisotropic Morawetz estimates). Consider $H=-\frac{1}{2} \Delta_{x}+V(x)-\frac{1}{2} \Delta_{y}$, where $V$ is a real-valued potential depending only on $x \in \mathbf{R}^{n}$. Let $\lambda>0$ and assume that the solution $u$ to (1.3) with $u_{\mid t=0}=u_{0} \in L^{2}\left(\mathbf{R}^{d}\right)$ exists globally, and satisfies

$$
\sup _{t \in \mathbf{R}}\left\|\nabla_{y} u(t)\right\|_{L^{2}\left(\mathbf{R}^{d}\right)}<\infty .
$$


Then we have

$$
\left\|\left|\nabla_{y}\right|^{\frac{3-(d-n)}{2}} R\right\|_{L_{t, y}^{2}\left(\mathbf{R} \times \mathbf{R}^{d-n}\right)}^{2} \leq C\left\|u_{0}\right\|_{L^{2}\left(\mathbf{R}^{d}\right)}^{3} \sup _{t \in \mathbf{R}}\left\|\nabla_{y} u(t)\right\|_{L^{2}\left(\mathbf{R}^{d}\right)}
$$

where

$$
R(t, y):=\int_{\mathbf{R}^{n}}|u(t, x, y)|^{2} d x,
$$

is the marginal of the mass density.

Using these estimates, we can prove asymptotic completeness results. Note however that since we consider energy-subcritical problems on $\mathbf{R}^{d}$, we assume

$$
\sigma<\frac{2}{(d-2)_{+}} .
$$

On the other hand, proving asymptotic completeness like in $\mathbf{R}^{d-n}$ via Morawetz estimates $(d-n$ is the "scattering dimension") is sensible, in view of the third point of Theorem 1.1 provided that

$$
\sigma>\frac{2}{d-n}
$$

The above two conditions are compatible if and only if $n=1$. This restriction is expected to be only technical, since scattering should occur under the weaker assumption

$$
\sigma>\frac{1}{d-n}
$$

but we lack of tools to decrease the value of $\sigma$ down to this threshold. Note that in the small data case, asymptotic completeness is established for $n=2$ provided that $d \geqslant 3$, for instance, from Theorem 1.5. On the other hand, it is our choice to state the result only for $d \leqslant 4$, since the case $d \geqslant 5$ would bring new technicalities in the presentation, but no new real difficulty (see also [32]).

Theorem 1.10 (Asymptotic completeness). Let $n=1,2 \leqslant d \leqslant 4$, and $\lambda>0$. Assume moreover:

- If $d=2: \sigma>2$.

- If $d=3: 1<\sigma<2$.

- If $d=4: 2 / 3<\sigma<1$.

For all $u_{0} \in \Sigma$, there exists a unique $u_{+} \in \Sigma$ such that the solution u provided by Proposition 1.4 satisfies

$$
\left\|e^{i t H} u(t)-u_{+}\right\|_{\Sigma} \underset{t \rightarrow+\infty}{\longrightarrow} 0 .
$$

1.5. Structure of the paper. In Section 2, we recall some of the aspects in [18, 31], with emphasis on aspects related to Strichartz estimates. In Section 3 , we propose a slight generalization of a result from [31], and establish global in time Strichartz estimates associated to $e^{-i t H}$ which are isotropic in space, in the same fashion as in [18], and as opposed to [32, 31]. These estimates allow us to prove Theorem 1.5 in Section 4 thanks to suitable vector-fields. Proposition 1.9 is established in Section 5 and Theorem 1.10, in Section 6 Finally in an appendix, we present formal arguments suggesting the existence of long range effects if $\sigma$ is not sufficiently large. 


\section{NONLINEAR SCHRÖDINGER EQUATION ON PRODUCT SPACES}

In this section, we recall some results which are highly related to the framework of the present paper, at least qualitatively.

A key step both in [31] and in [18] consists in proving global in time Strichartz estimates, even though the free dynamics is not fully dispersive due to the boundedness of the compact manifold ( $\mathbf{T}^{2}$ in the case of [18]). For $M^{n}$ an $n$-dimensional compact manifold without boundary, consider

$$
i \partial_{t} u+\frac{1}{2} \Delta_{M^{n} \times \mathbf{R}^{d-n}} u=F .
$$

We introduce once and for all the notion of admissible pairs inspired by Strichartz estimates on $\mathbf{R}^{k}$. The definition includes a notion of "dispersive dimension", and will be of constant use in this paper.

Definition 2.1. Let $k$ be a positive integer. A pair $(q, r)$ is $k$-admissible if $2 \leqslant r<\frac{2 k}{k-2}$ $(2 \leqslant r \leqslant \infty$ if $k=1,2 \leqslant r<\infty$ if $k=2)$ and

$$
\frac{2}{q}=k\left(\frac{1}{2}-\frac{1}{r}\right) \text {. }
$$

Proposition 2.2 (From [31]). Let $d \geqslant 2,1 \leqslant n \leqslant d-1$ and $M^{n}$ be an $n$-dimensional compact manifold without boundary. For $(x, y) \in M^{n} \times \mathbf{R}^{d-n}$, the following estimate holds: there exists $C_{r_{1}, r_{2}}$ such that

$$
\begin{aligned}
\left\|e^{i t \Delta_{x, y}} f\right\|_{L_{t}^{p_{1}} L_{y}^{r_{1}} L_{x}^{2}}+ & \left\|\int_{0}^{t} e^{i(t-s) \Delta_{x, y}} F(s, x, y) d s\right\|_{L_{t}^{p_{1}} L_{y}^{r_{1}} L_{x}^{2}} \\
& \leqslant C_{r_{1}, r_{2}}\left(\|f\|_{L_{x, y}^{2}}+\|F\|_{L_{t}^{p_{2}^{\prime}} L_{y}^{r_{2}^{\prime}} L_{x}^{2}}\right),
\end{aligned}
$$

where the pairs $\left(p_{1}, r_{1}\right)$ and $\left(p_{2}, r_{2}\right)$ are $(d-n)$-admissible.

The proof in [31] relies on the fact that $\Delta_{M^{n}}$ possesses an eigenbasis in $L^{2}\left(M^{n}\right)$ : by decomposing any solution to 2.1 on this eigenbasis, each coefficient solves a Schrödinger equation on $\mathbf{R}^{d-n}$, and satisfies global Strichartz estimates. Proposition 2.2 follows by summing these inequalities in $L^{2}\left(M^{n}\right)$ and invoking Minkowski inequality. In Section 3.1 we present a generalization of this result, which does not require any spectral analysis.

In the case where $d=3, n=2$ and $M^{2}=\mathbf{T}^{2}$, another family of estimates has been established in [18]. For $\gamma \in \mathbf{Z}$, set $I_{\gamma}=2 \pi[\gamma, \gamma+1)$.

Proposition 2.3 (From [18]). Let $N \geqslant 1$ be dyadic, then

$$
\left\|e^{i t \Delta_{\mathbf{R} \times \mathbf{T}^{2}} P} \leqslant N u_{0}\right\|_{\ell_{\gamma}^{q} L_{t, x, y}^{p}\left(I_{\gamma} \times \mathbf{R} \times \mathbf{T}^{2}\right)} \lesssim N^{\left(\frac{3}{2}-\frac{5}{p}\right)}\left\|u_{0}\right\|_{L^{2}\left(\mathbf{R} \times \mathbf{T}^{2}\right)},
$$

whenever

$$
p>4 \text { and } \frac{2}{q}+\frac{1}{p}=\frac{1}{2},
$$

and where $P_{\leqslant N}$ stands for a frequency cut-off.

An important difference with the approach in [31] is that in the case $M^{n}=\mathbf{T}^{n}$, the eigenbasis of the Laplacian possesses a group structure, which makes it possible to proceed with more explicit computations. Extensive use of Strichartz estimates on $\mathbf{T}^{2}$ [4] (see also [13]) is also made to prove Proposition 2.3. We emphasize however that the proof of Proposition 2.3 is rather involved technically. We prove an analogous result in the framework of 1.3 in Section 3.2 Even though the group structure of the eigenfunctions 
of $H$ is lost (the eigenfunctions are given by Hermite functions), computations are less involved, and one does not have to face a loss of regularity for local Strichartz estimates associated to the harmonic oscillator.

\section{Global in time Strichartz estimates}

3.1. A slight generalization. In this subsection, we generalize Proposition 2.2 in a case where the existence of an eigenbasis for the operator in the $x$ variable is not assumed.

Proposition 3.1. Let $d \geqslant 2,1 \leqslant n \leqslant d-1, X^{n}$ be an $n$-dimensional space, and $y \in \mathbf{R}^{d-n}$. Let $P$ be an operator acting on the $x$ variable, but not on the $y$ variable. In particular,

$$
\left[P, \Delta_{y}\right]=0
$$

Assume that the flow generated by $P$ is uniformly bounded on $L^{2}\left(X^{n}\right)$, at least in the future,

$$
\exists C>0, \quad\left\|e^{i t P} v_{0}\right\|_{L^{2}\left(X^{n}\right)} \leqslant C\left\|v_{0}\right\|_{L^{2}\left(X^{n}\right)}, \quad \forall t \geqslant 0 .
$$

Then for all $u_{0} \in L^{2}\left(\mathbf{R}^{d}\right)$, all $(d-n)$-admissible pairs $\left(p_{1}, r_{1}\right)$ and $\left(p_{2}, r_{2}\right)$, there exists $C_{r_{1}, r_{2}}$ such that the solution to

$$
i \partial_{t} u+P u+\Delta_{y} u=F, \quad u_{\mid t=0}=u_{0}
$$

satisfies:

$$
\|u\|_{L_{t}^{p_{1}}\left(\mathbf{R}_{+} ; L_{y}^{r_{1}} L_{x}^{2}\right)} \leqslant C_{r_{1}, r_{2}}\left(\left\|u_{0}\right\|_{L_{x, y}^{2}}+\|F\|_{L_{t}^{p_{2}^{\prime}\left(\mathbf{R}_{+} ; L_{y}^{r_{2}^{\prime}} L_{x}^{2}\right)}}\right) .
$$

For instance, $X^{n}$ may be any manifold without boundary, and $P$ a Schrödinger operator, including a real-valued external potential or magnetic field, with decay or sign assumptions to ensure that the flow is well-defined on $L^{2}\left(X^{n}\right)$. In this cases, it may happen that no spectral theory like in [31] is available. In general, the $X^{n}$ part can be viewed as a black box, and Proposition 3.1 is a way to take advantage of the Schrödinger dispersion on $\mathbf{R}^{d-n}$.

Example 3.2. In the case of Schrödinger operators on $\mathbf{R}^{n}, P=-\Delta_{x}+V(x)$, it suffices to consider $V=V_{1}+V_{2}$, with (see [30, p. 199]) $V_{1}, V_{2}$ real-valued and measurable, and

- $V_{1}(x) \geqslant-a|x|^{2}-b$ for some constants $a$ and $b$.

- $V_{2} \in L^{p}\left(\mathbf{R}^{n}\right)$ with $p \geqslant 2$ if $n \leqslant 3, p>2$ if $n=4$, and $p \geqslant n / 2$ if $n \geqslant 5$.

This includes in particular the case studied in this paper, $V(x)=|x|^{2} / 2$.

Example 3.3. Still on $\mathbf{R}^{n}, P$ may be any polynomial in $D_{x}=-i \partial_{x}$, not necessarily elliptic, or more generally a real-valued Fourier multiplier.

Proof. The commutation assumption (3.1) implies

$$
e^{i t\left(P+\Delta_{y}\right)}=e^{i t P} e^{i t \Delta_{y}}=e^{i t \Delta_{y}} e^{i t P} .
$$

Using this remark, the assumption that $e^{i t P}$ is bounded on $L^{2}\left(X^{n}\right)$ for positive time, and the standard properties of the Schrödinger group on $\mathbf{R}^{d-n}$, we have:

$$
\left\|e^{i t\left(P+\Delta_{y}\right)}\right\|_{L_{x, y}^{2} \rightarrow L_{x, y}^{2}} \lesssim 1, \quad\left\|e^{i t\left(P+\Delta_{y}\right)}\right\|_{L_{y}^{1} L_{x}^{2} \rightarrow L_{y}^{\infty} L_{x}^{2}} \lesssim \frac{1}{t^{(d-n) / 2}}, \quad t>0 .
$$

Invoking [21, Theorem 10.1], with

$$
B_{0}=H=L_{x, y}^{2}, \quad B_{1}=L_{y}^{1} L_{x}^{2}
$$


we have

$$
\begin{aligned}
& \left\|e^{i t\left(P+\Delta_{y}\right)} u_{0}\right\|_{L_{t}^{p}\left(\mathbf{R}_{+} ; B_{\theta}^{*}\right)} \lesssim\left\|u_{0}\right\|_{H}, \\
& \left\|\int_{0}^{t} e^{i(t-s)\left(P+\Delta_{y}\right)} F(s) d s\right\|_{L_{t}^{p_{1}}\left(\mathbf{R}_{+} ; B_{\theta_{1}}^{*}\right)} \lesssim\|F\|_{L_{t}^{p_{2}^{\prime}}\left(\mathbf{R}_{+} ; B_{\theta_{2}}\right)},
\end{aligned}
$$

where for $0 \leqslant \theta \leqslant 1, B_{\theta}$ denotes the real interpolation space $\left(B_{0}, B_{1}\right)_{\theta, 2}$, and $2 / p=$ $(d-n) \theta / 2, p \geqslant 2,(p, \theta, d-n) \neq(2,1,1)$, hence the proposition.

3.2. The case of partial harmonic confinement. We split the Hamiltonian $H$ into two parts, the confining one and the fully dispersive one, by denoting

$$
H_{1}=-\frac{1}{2} \Delta_{x}+\frac{|x|^{2}}{2} ; \quad H_{2}=-\frac{1}{2} \Delta_{y} .
$$

As in the previous subsection, we note that both operators commute, $\left[H_{1}, H_{2}\right]=0$, therefore their corresponding propagators also commute

$$
e^{-i t H}=e^{-i t\left(H_{1}+H_{2}\right)}=e^{-i t H_{1}} e^{-i t H_{2}}=e^{-i t H_{2}} e^{-i t H_{1}} .
$$

The classical formula

$$
e^{-i t H_{2}} g(y)=\frac{1}{(2 i \pi t)^{(d-n) / 2}} \int_{\mathbf{R}^{d-n}} e^{i \frac{\left|y-y^{\prime}\right|^{2}}{2 t}} f\left(y^{\prime}\right) d y^{\prime}
$$

yields the standard global dispersive estimate

$$
\left\|e^{-i t H_{2}}\right\|_{L^{1}\left(\mathbf{R}^{d-n}\right) \rightarrow L^{\infty}\left(\mathbf{R}^{d-n}\right)} \leqslant \frac{1}{(2 \pi|t|)^{(d-n) / 2}}, \quad \forall t \neq 0 .
$$

Similarly, the fundamental solution associated to $H_{1}$ is given by Mehler's formula [25]:

$$
e^{-i t H_{1}} f(x)=\frac{1}{(2 i \pi \sin t)^{n / 2}} \int_{\mathbf{R}^{n}} e^{\frac{i}{2 \sin t}\left(\left(|x|^{2}+\left|x^{\prime}\right|^{2}\right) \cos t-2 x \cdot x^{\prime}\right)} f\left(x^{\prime}\right) d x^{\prime},
$$

where the square root of sin $t$ means implicitly that the singularities of the fundamental solution are taken into account: every time $\sin t=0$, a phase shift appears (Maslov index; see e.g. [20, 34]). The only information that we shall actually use is that local in time dispersive estimates are available, periodically in time:

$$
\left\|e^{-i t H_{1}}\right\|_{L^{1}\left(\mathbf{R}^{n}\right) \rightarrow L^{\infty}\left(\mathbf{R}^{n}\right)} \leqslant \frac{1}{(2 \pi|\sin t|)^{n / 2}}, \quad \forall t \notin \pi \mathbf{Z} .
$$

A property that we will use crucially to establish global Strichartz estimates for $e^{-i t H}$ is the fact that the above right hand side is periodic (see Remark 3.5 below). Accordingly, for $\gamma \in \mathbf{Z}$, we set $I_{\gamma}=\pi[\gamma-1, \gamma+1)$.

Theorem 3.4 (Global Strichartz estimates). Let $d \geqslant 2$ and $1 \leqslant n \leqslant d-1$. If $2 \leqslant r<\frac{2 d}{d-2}$ and the pairs $(q, r)$ and $(p, r)$ are, respectively, $d$-admissible and $(d-n)$-admissible, then the following two inequalities hold,

$$
\left\|e^{-i t H} u_{0}\right\|_{\ell_{\gamma}^{p} L^{q}\left(I_{\gamma} ; L^{r}\left(\mathbf{R}^{d}\right)\right)} \lesssim\left\|u_{0}\right\|_{L^{2}\left(\mathbf{R}^{d}\right)}
$$

and

$$
\left\|\int_{t \in \mathbf{R}} e^{i t H} F(t) d t\right\|_{L^{2}\left(\mathbf{R}^{d}\right)} \lesssim\|F\|_{\ell_{\gamma}^{p^{\prime}} L^{q^{\prime}}\left(I_{\gamma} ; L^{r^{\prime}}\left(\mathbf{R}^{d}\right)\right)} .
$$


Also, if $\left(p_{1}, q_{1}, r_{1}\right)$ and $\left(p_{2}, q_{2}, r_{2}\right)$ are two such triplets, then

$$
\left\|\int_{0}^{t} e^{-i(t-s) H} F(s) d s\right\|_{\ell_{\gamma}^{p_{1} L^{q_{1}}\left(I_{\gamma} ; L^{r_{1}}\left(\mathbf{R}^{d}\right)\right)}} \lesssim\|F\|_{\ell_{\gamma}^{p_{2}^{\prime} L_{2}^{q_{2}^{\prime}}\left(I_{\gamma} ; L^{r_{2}^{\prime}}\left(\mathbf{R}^{d}\right)\right)}} .
$$

The above statement can be understood as follows. Locally in time, we have the same Strichartz estimates as for $e^{i t \Delta}$, a fact that is standard in the presence of a smooth potential growing at most quadratically in space (see e.g. [9]). In the present case, these estimates are made global in time thanks to the dispersion in the $y$ directions, corresponding to the free Hamiltonian $\mathrm{H}_{2}$ : there are $d-n$ such directions, so the assumption for $(p, r)$ to be $(d-n)$-admissible appears very natural.

Proof. The scheme of this proof follows the same general method as for standard Strichartz inequalities, by using duality and the $T T^{*}$ argument of Tomas-Stein. The added novelty here is strongly inspired by the arguments in [10, 18].

The homogeneous inequalities (3.4) and (3.5) are dual to each other and therefore equivalent.

We now prove (3.5). Let $F, G \in C^{\infty}\left(\mathbf{R} \times \mathbf{R}^{d}\right)$. Then, (3.5) is equivalent to the bilinear inequality

$$
\begin{aligned}
\left|\left\langle\int_{t \in \mathbf{R}} e^{i t H} F(t) d t, \int_{s \in \mathbf{R}} e^{i s H} G(s) d s\right\rangle_{L^{2}}\right| & \\
& \lesssim\|F\|_{\ell_{\gamma}^{p^{\prime}} L^{q^{\prime}}\left(I_{\gamma} ; L^{r^{\prime}}\left(\mathbf{R}^{d}\right)\right)}\|G\|_{\ell_{\gamma}^{p^{\prime}} L^{q^{\prime}}\left(I_{\gamma} ; L^{r^{\prime}}\left(\mathbf{R}^{d}\right)\right)},
\end{aligned}
$$

which, again by duality, is in turn equivalent to

$$
\left\|\int_{s \in \mathbf{R}} e^{-i(t-s) H} G(s) d s\right\|_{\ell_{\gamma}^{p} L^{q}\left(I_{\gamma} ; L^{r}\left(\mathbf{R}^{d}\right)\right)} \lesssim\|G\|_{\ell_{\gamma}^{p^{\prime}} L^{q^{\prime}}\left(I_{\gamma} ; L^{r^{\prime}}\left(\mathbf{R}^{d}\right)\right)} .
$$

To prove (3.7) we use a partition of unity in time

$$
\sum_{\gamma \in \mathbf{Z}} \chi(t-\pi \gamma)=1, \quad \forall t \in \mathbf{R} \quad \text { with } \quad \operatorname{supp} \chi \subset[-\pi, \pi] .
$$

We thus have

$$
\begin{aligned}
&\left\langle\int_{t \in \mathbf{R}} e^{i t H} F(t) d t, \int_{s \in \mathbf{R}} e^{i s H} G(s) d s\right\rangle_{L^{2}} \\
&=\sum_{\alpha, \beta \in \mathbf{Z}} \iint_{t, s \in \mathbf{R}}\left\langle\chi(t-\pi \alpha) e^{i t H} F(t), \chi(t-\pi \beta) e^{i s H} G(s)\right\rangle_{L^{2}} d t d s .
\end{aligned}
$$

This sum is now bounded by splitting it into the diagonal terms, $|\alpha-\beta| \leqslant 1$, and the non-diagonal ones, $|\alpha-\beta|>1$.

The sum of the diagonal terms is bounded as follows

$$
\begin{aligned}
& \sum_{|\alpha-\beta| \leqslant 1}\left|\iint_{t, s \in \mathbf{R}}\left\langle\chi(t-\pi \alpha) e^{i t H} F(t), \chi(t-\pi \beta) e^{i s H} G(s)\right\rangle_{L^{2}} d t d s\right| \\
& \quad \lesssim \sum_{|\alpha-\beta| \leqslant 1}\left\|\int_{-\pi}^{\pi} \chi(t) e^{i(t+\pi \alpha) H} F(t+\pi \alpha) d t\right\|\left\|_{L^{2}}\right\| \int_{-\pi}^{\pi} \chi(s) e^{i(s+\pi \beta) H} G(s+\pi \beta) d s \|_{L^{2}} \\
& \quad=\sum_{|\alpha-\beta| \leqslant 1}\left\|\int_{-\pi}^{\pi} \chi(t) e^{i t H} F(t+\pi \alpha) d t\right\|_{L^{2}}\left\|\int_{-\pi}^{\pi} \chi(s) e^{i s H} G(s+\pi \beta) d s\right\|_{L^{2}},
\end{aligned}
$$


where, in this last step, we have used the fact that the propagator $e^{-i t H}$ is unitary in $L^{2}$. At this point, we use Strichartz estimates in their dual form (analogous to (3.5), which are available locally in time, for the partially confined operator $H$, with $d$-admissible pairs $(q, r)$

$$
\begin{aligned}
& \lesssim \sum_{|\alpha-\beta| \leqslant 1}\|F(\cdot+\pi \alpha)\|_{L^{q^{\prime}}\left([-\pi, \pi] ; L^{r^{\prime}}\left(\mathbf{R}^{d}\right)\right)}\|G(\cdot+\pi \beta)\|_{L^{q^{\prime}}\left([-\pi, \pi] ; L^{r^{\prime}}\left(\mathbf{R}^{d}\right)\right)} \\
& \lesssim\|F\|_{\ell_{\gamma}^{2} L^{q^{\prime}}\left(I_{\gamma} ; L^{r^{\prime}}\left(\mathbf{R}^{d}\right)\right)}\|G\|_{\ell_{\gamma}^{2} L^{q^{\prime}}\left(I_{\gamma} ; L^{r^{\prime}}\left(\mathbf{R}^{d}\right)\right)} .
\end{aligned}
$$

For the non-diagonal part of the sum - the crucial and deeper one - we proceed with a different approach, involving a localized $T T^{*}$ argument.

$$
\begin{aligned}
& \sum_{|\alpha-\beta|>1}\left|\iint_{t, s \in \mathbf{R}}\left\langle\chi(t-\pi \alpha) e^{i t H} F(t), \chi(t-\pi \beta) e^{i s H} G(s)\right\rangle_{L^{2}} d t d s\right| \\
& =\sum_{|\alpha-\beta|>1}\left|\iint_{t, s \in \mathbf{R}} \chi(t) \chi(s)\left\langle F(t+\pi \alpha), e^{-i(t-s+\pi(\alpha-\beta)) H} G(s+\pi \beta)\right\rangle_{L^{2}} d t d s\right| \\
& \quad \lesssim \sum_{\substack{\beta \in \mathbf{Z} \\
|\gamma|>1}}\left|\iint_{(t, s) \in[-\pi, \pi]^{2}} \chi(t) \chi(s)\left\langle F(t+\pi(\beta+\gamma)), e^{-i(t-s+\pi \gamma) H} G(s+\pi \beta)\right\rangle_{L^{2}} d t d s\right| \\
& \quad \lesssim \sum_{\substack{\beta \in \mathbf{Z} \\
|\gamma|>1}} \iint_{(t, s) \in[-\pi, \pi]^{2}}\|F(t+\pi(\beta+\gamma))\|_{L^{r^{\prime}}}\left\|e^{-i(t-s+\pi \gamma) H} G(s+\pi \beta)\right\|_{L^{r}} .
\end{aligned}
$$

We have now reached the stage where we use the time decay estimate of the dispersive propagator. For that, we exploit the commutativity property of the partial propagators $e^{-i t H_{1}}$ and $e^{-i t H_{2}}$, as well as the periodicity in time of $e^{-i t H_{1}}$.

Thus, following the remarks at the beginning of this section, we know that

$$
e^{-i(t-s+\pi \gamma) H}=e^{-i(t-s+\pi \gamma) H_{1}} e^{-i(t-s+\pi \gamma) H_{2}} .
$$

The propagator for $\mathrm{H}_{2}$ corresponds to the free Schrödinger case in the $y$ variables, thus has a global time decay with rate $t^{-(d-n) / 2}$, as seen in (3.2). Whereas the propagator for $H_{1}$ corresponds to the time periodic harmonic oscillator in the $x$ variables, with local in time decay with rate $t^{-n / 2}$ from 3.3 . So, doing the full $L^{1}\left(\mathbf{R}^{d}\right) \rightarrow L^{\infty}\left(\mathbf{R}^{d}\right)$ estimate for the propagator of $H$ by first splitting the variables $x$ and $y$, then applying the partial propagators one at a time for each of the corresponding variables and using the periodicity of $H_{1}$, we obtain the combined time decay rate

$$
\left\|e^{-i(t-s+\pi \gamma) H} f\right\|_{L_{x, y}^{\infty}\left(\mathbf{R}^{d}\right)} \lesssim \frac{1}{|t-s|^{n / 2}} \frac{1}{|\gamma|^{(d-n) / 2}}\|f\|_{L_{x, y}^{1}\left(\mathbf{R}^{d}\right)},
$$

which is local in the $t, s$ variables (from the partially confining operator $H_{1}$ acting on the $x$ variables) and global in $\gamma$ (from the free operator $H_{2}$ acting on the $y$ variables). Finally, interpolating the above $L^{1} \rightarrow L^{\infty}$ estimate with the $L^{2}$ conservation, we obtain the intermediate $L^{r^{\prime}} \rightarrow L^{r}$ decay to be used within the $T T^{*}$ proof of the Strichartz estimates,

$$
\left\|e^{-i(t-s+\pi \gamma) H} f\right\|_{L_{x, y}^{r}\left(\mathbf{R}^{d}\right)} \lesssim \frac{1}{|t-s|^{n(1 / 2-1 / r)}} \frac{1}{|\gamma|^{(d-n)(1 / 2-1 / r)}}\|f\|_{L_{x, y}^{r^{\prime}}\left(\mathbf{R}^{d}\right)},
$$

for $2 \leqslant r \leqslant \infty$. 
So, plugging this estimate back into the non-diagonal sum inequality, we thus obtain

$$
\begin{aligned}
& \lesssim \sum_{\substack{\beta \in \mathbf{Z} \\
|\gamma|>1}} \iint_{(t, s) \in[-\pi, \pi]^{2}}\|F(t+\pi(\beta+\gamma))\|_{L^{r^{\prime}}} \frac{1}{|t-s|^{n\left(\frac{1}{2}-\frac{1}{r}\right)}} \frac{1}{|\gamma|^{(d-n)\left(\frac{1}{2}-\frac{1}{r}\right)}}\|G(s+\pi \beta)\|_{L^{r^{\prime}}} \\
& \lesssim \sum_{\substack{\beta \in \mathbf{Z} \\
|\gamma|>1}} \frac{1}{|\gamma|^{(d-n)\left(\frac{1}{2}-\frac{1}{r}\right)}} \iint_{(t, s) \in[-\pi, \pi]^{2}} \frac{1}{|t-s|^{n\left(\frac{1}{2}-\frac{1}{r}\right)}}\|F(t+\pi(\beta+\gamma))\|_{L^{r^{\prime}}}\|G(s+\pi \beta)\|_{L^{r^{\prime}}} \\
& \lesssim \sum_{\substack{\beta \in \mathbf{Z} \\
|\gamma|>1}} \frac{1}{|\gamma|^{(d-n)\left(\frac{1}{2}-\frac{1}{r}\right)}}\|F(\cdot+\pi(\beta+\gamma))\|_{L^{q^{\prime}}\left([-\pi, \pi] ; L^{r^{\prime}}\left(\mathbf{R}^{d}\right)\right)}\|G(\cdot+\pi \beta)\|_{L^{q^{\prime}\left([-\pi, \pi] ; L^{r^{\prime}}\left(\mathbf{R}^{d}\right)\right)},},
\end{aligned}
$$

where we have used the Hardy-Littlewood-Sobolev inequality in the $t, s$ variables, in the last step, for $(q, r)$ now an $n$-admissible pair. To conclude this estimate, we again apply the Hardy-Littlewood-Sobolev inequality, this time in its discrete version for the $\gamma$ variable, to obtain

$$
\lesssim\|F\|_{\ell_{\gamma}^{p^{\prime}} L^{q^{\prime}}\left(I_{\gamma} ; L^{r^{\prime}}\left(\mathbf{R}^{d}\right)\right)}\|G\|_{\ell_{\gamma}^{p^{\prime}} L^{q^{\prime}}\left(I_{\gamma} ; L^{r^{\prime}}\left(\mathbf{R}^{d}\right)\right)}
$$

whenever

$$
\frac{2}{p} \leqslant(d-n)\left(\frac{1}{2}-\frac{1}{r}\right),
$$

in particular for $(p, r)$ a $(d-n)$-admissible pair.

To finish the proof of (3.7) we gather the diagonal and non-diagonal estimates, (3.9) and (3.10), to obtain

$$
\begin{aligned}
& \left|\left\langle\int_{t \in \mathbf{R}} e^{i t H} F(t) d t, \int_{s \in \mathbf{R}} e^{i s H} G(s) d s\right\rangle_{L^{2}}\right|
\end{aligned}
$$

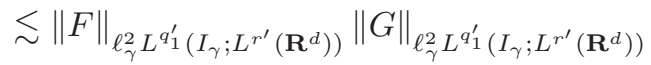

$$
\begin{aligned}
& +\|F\|_{\ell_{\gamma}^{p^{\prime}} L^{q_{2}^{\prime}}\left(I_{\gamma} ; L^{r^{\prime}}\left(\mathbf{R}^{d}\right)\right)}\|G\|_{\ell_{\gamma}^{p^{\prime}} L^{q_{2}^{\prime}}\left(I_{\gamma} ; L^{r^{\prime}}\left(\mathbf{R}^{d}\right)\right)},
\end{aligned}
$$

where the pair $\left(q_{1}, r\right)$ is $d$-admissible, while $\left(q_{2}, r\right)$ is $n$-admissible. Therefore $q_{2}^{\prime}<q_{1}^{\prime}$ and using the inclusion $L^{q_{1}^{\prime}}\left(I_{\gamma}\right) \subset L^{q_{2}^{\prime}}\left(I_{\gamma}\right)$, while $\ell^{p^{\prime}} \subset \ell^{2}$, we obtain 3.7. Thus, we have established (3.4) and (3.5), as well as (3.8).

There still remains establishing 3.6 to conclude the proof of the theorem. But this finite interval version follows from the similar global integral estimate (3.8) by using the now standard Christ-Kiselev lemma, together with interpolation between admissible triplets, just as in the analogous final stage of a classical Strichartz estimate proof.

Remark 3.5 (More general potentials). The two properties of $e^{-i t H_{1}}$ that we have used in the proof of Theorem 3.4 are the existence of local dispersive estimates, and the fact that such estimates are available periodically in time. The first point remains for smooth potentials which grow at most quadratically, from [12]. The fact that these dispersive estimates are available periodically in time remains for instance when the harmonic potential is suitably perturbed with a smooth one (Schwartz class), so the new operator has the same spectrum as the harmonic oscillator; this has been established in the case $n=1$ in [24]. Also, we may consider more general quadratic potentials, when $n \geqslant 2$,

$$
\sum_{j=1}^{n} \omega_{j}^{2} \frac{x_{j}^{2}}{2}, \quad \omega_{j}>0 .
$$


If the $\omega_{j}$ 's are rationally dependent, then the above proof can easily be repeated, since local in time dispersive estimates remain, as well as time periodicity of the propagator (each component leads to dispersive estimates which are $\pi / \omega_{j}$-periodic in time). On the other hand, if the $\omega_{j}$ 's are rationally independent, either by using Hermite functions (eigen decomposition) or by invoking Mehler's formula, we see that the time periodicity is lost. The argument that we have followed to obtain global Strichartz estimates no longer works; the dynamics might be rather involved, typically due to the presence of small divisors. On the other hand, since Proposition 3.1 only assumes that the flow associated to the operator $P\left(=\frac{1}{2} \Delta_{x}+V(x)\right.$ here $)$, is bounded on $L^{2}$, its conclusion remains valid for all quadratic potentials.

\section{EXISTENCE OF WAVE OPERATORS AND SMALL DATA SCATTERING}

In this section, we prove Theorem 1.5. The existence of wave operators is obtained by a fixed point argument in a suitable space, constructed in view of global Strichartz estimates provided by Theorem 3.4. Asymptotic completeness for small data follows essentially from the same estimates, plus a standard bootstrap argument.

4.1. Vector fields. As pointed out in the introduction, proving the existence of wave operator in $\Sigma$ in the absence of external potential, that is for (1.4), relies on the use of the vector field $x+i t \nabla$. In the case of $(1.3)$, this means that we consider

$$
J=y+i t \nabla_{y}=i t e^{i|y|^{2} /(2 t)} \nabla_{y}\left(e^{-i|y|^{2} /(2 t)}\right) .
$$

In view of the second identity, $J$ acts on the gauge invariant nonlinearity $|u|^{2 \sigma} u$ like $\nabla_{y}$, and Gagliardo-Nirenberg inequalities involving $\nabla_{y}$ export to similar inequalities in terms of $J$, with an extra time dependent factor:

$$
\|f\|_{L^{r}\left(\mathbf{R}^{d}\right)} \leqslant \frac{C_{r}}{|t|^{(d-n)(1 / 2-1 / r)}}\|f\|_{L^{2}\left(\mathbf{R}^{d}\right)}^{1-d(1 / 2-1 / r)}\left\|\left(\nabla_{x}, J\right) f\right\|_{L^{2}\left(\mathbf{R}^{d}\right)}^{d(1 / 2-1 / r)},
$$

for all $2 \leqslant r \leqslant \frac{2 d}{d-2}(2 \leqslant r<\infty$ if $d=2)$. Finally, $J$ commutes with the linear part of (1.3), $\left[i \partial_{t}-H, J\right]=0$.

We now turn to the $x$-dependent part of $H$. In view of the above weighted GagliardoNirenberg inequality, we will get the same time decay as on $\mathbf{R}^{d-n}$ provided that we can estimate $\nabla_{x} u$ (in $L^{2}$ ). However, unlike $J, \nabla_{x}$ does not commute with the linear part of (1.3):

We remark that

$$
\left[i \partial_{t}-H, \nabla_{x}\right]=-x
$$

$$
\left[i \partial_{t}-H, x\right]=\nabla_{x},
$$

so it is possible to obtain a closed system of estimates for $\left(u, J u, \nabla_{x} u, x u\right)$. This remark can be used to construct a solution to (1.3) locally in time, thanks to the Gronwall lemma; see [26] (see also [5]). However, the above commutators generate an exponentially growing factor after the use of the Gronwall lemma, an aspect which ruins the algebraic time decay provided by (4.1) when large time is considered, like in scattering theory. Therefore, we need to replace the operators $\nabla_{x}$ and $x$ by adapted operators which enjoy properties similar to those of $J$. In the case of the harmonic potential, such operators are available: see e.g. [5]. We introduce

$$
\begin{array}{ll}
A_{1}(t)=x \sin t-i \cos t \nabla_{x}, & A_{2}(t)=x \cos t+i \sin t \nabla_{x} \\
A_{3}(t)=-i \nabla_{y}, & A_{4}(t)=y+i t \nabla_{y} .
\end{array}
$$


To ease the forthcoming formulas, we also set

$$
A_{0}=\text { Id. }
$$

The operators $A_{3}$ and $A_{4}$ are the standard ones in the absence of external potential. The operators $A_{1}$ and $A_{2}$ are adapted to the harmonic potential, and satisfy

$$
\left(\begin{array}{c}
A_{1}(t) \\
-A_{2}(t)
\end{array}\right)=\left(\begin{array}{cc}
\sin t & \cos t \\
-\cos t & \sin t
\end{array}\right)\left(\begin{array}{c}
x \\
-i \nabla_{x}
\end{array}\right) \text {. }
$$

Indeed, the operators $A_{1}$ and $A_{2}$ account for the fact that the harmonic oscillator rotates the phase space at a speed which is uniform in $(x, \xi)$. In particular, we have the pointwise identity for, say, $f \in \mathcal{S}\left(\mathbf{R}^{d}\right)$,

$$
\left|A_{1}(t) f(x, y)\right|^{2}+\left|A_{2}(t) f(x, y)\right|^{2}=|x f(x, y)|^{2}+\left|\nabla_{x} f(x, y)\right|^{2}, \quad \forall t \in \mathbf{R} .
$$

Lemma 4.1. For $1 \leqslant j \leqslant 4$, the operators $A_{j}$ satisfy the following properties.

- They correspond to the conjugation of gradient and momentum by the free flow,

$$
\begin{array}{ll}
A_{1}(t)=e^{-i t H}\left(-i \nabla_{x}\right) e^{i t H}, & A_{2}(t)=e^{-i t H} x e^{i t H}, \\
A_{3}(t)=e^{-i t H}\left(-i \nabla_{y}\right) e^{i t H}, & A_{4}(t)=e^{-i t H} y e^{i t H} .
\end{array}
$$

Therefore, they commute with the linear part of $(1.3)$ : $\left[i \partial_{t}-H, A_{j}(t)\right]=0$.

- They act on gauge invariant nonlinearities like derivatives. In particular, we have the pointwise estimate

$$
\left|A_{j}(t)\left(|u|^{2 \sigma} u\right)\right| \lesssim|u|^{2 \sigma}\left|A_{j}(t) u\right| .
$$

- Weighted Gagliardo-Nirenberg inequalities: for all $2 \leqslant r \leqslant \frac{2 d}{d-2}(2 \leqslant r<\infty$ if $d=2$ ), there exists $K_{r}$ such that for all $f \in \Sigma, t \neq 0$,

$$
\|f\|_{L^{r}\left(\mathbf{R}^{d}\right)} \leqslant \frac{K_{r}}{\langle t\rangle^{(d-n)(1 / 2-1 / r)}}\|f\|_{L^{2}\left(\mathbf{R}^{d}\right)}^{1-\delta}\left(\sum_{j=1}^{4}\left\|A_{j}(t) f\right\|_{L^{2}\left(\mathbf{R}^{d}\right)}\right)^{\delta}
$$

with $\delta=d(1 / 2-1 / r)$.

The last two points follow directly from the "nonlinear" counterpart of the "linear" factorization of the first point:

$$
\begin{aligned}
& A_{1}(t) f=-i \cos t e^{-\frac{i}{2}|x|^{2} \tan t} \nabla_{x}\left(e^{\frac{i}{2}|x|^{2} \tan t} f\right), \\
& A_{2}(t) f=i \sin t e^{i|x|^{2} /(2 \tan t)} \nabla_{x}\left(e^{-i|x|^{2} /(2 \tan t)} f\right), \\
& A_{4}(t) f=i t e^{i|y|^{2} /(2 t)} \nabla_{y}\left(e^{-i|y|^{2} /(2 t)} f\right) .
\end{aligned}
$$

We emphasize that analogous operators are available for anisotropic quadratic potentials. On the other hand, the existence of operators enjoying the above three properties seems to be bound to potentials which are polynomials of degree at most two ([8]).

In view of the first point in Lemma4.1 we have the equivalence, in the sense of equivalence of norms:

$$
\begin{aligned}
\left\|e^{i t H} u(t)-u_{-}\right\|_{\Sigma} & \sim \sum_{j=0}^{4}\left\|A_{j}(t) u(t)-A_{j}(t) e^{-i t H} u_{-}\right\|_{L^{2}} \\
& \sim \sum_{j=0}^{4}\left\|A_{j}(t) u(t)-e^{-i t H} A_{j}(0) u_{-}\right\|_{L^{2}} .
\end{aligned}
$$


4.2. The fixed point argument. Let $u_{-} \in \Sigma$. For $\gamma_{0} \leqslant-1$, we denote

$$
J_{\gamma_{0}}=\left(-\infty, \pi\left(\gamma_{0}+1\right)\right)=\bigcup_{\gamma \leqslant \gamma_{0}} I_{\gamma}
$$

and we set

$$
\begin{aligned}
& X_{\gamma_{0}}=\left\{u \in L^{\infty}\left(J_{\gamma_{0}} ; H^{1}\left(\mathbf{R}^{d}\right)\right) ; \text { for all } j \in\{0, \ldots, 4\},\right. \\
& \left.\quad\left\|A_{j} u\right\|_{L^{\infty}\left(J_{\gamma_{0}} ; L^{2}\right)} \leqslant 2\left\|A_{j}(0) u_{-}\right\|_{L^{2}}, \quad\left\|A_{j} u\right\|_{\ell_{\gamma \leqslant \gamma_{0}}^{p} L^{q}\left(I_{\gamma} ; L^{r}\right)} \leqslant 2 C_{r}\left\|A_{j}(0) u_{-}\right\|_{L^{2}}\right\},
\end{aligned}
$$

where $C_{r}$ stems from Theorem 3.4. The indices $p, q, r$ are precised below: in view of Theorem 3.4, they are such that $(q, r)$ is $d$-admissible, and $(p, r)$ is $(d-n)$-admissible. Theorem 1.5 follows from a fixed point argument on Duhamel's formula

$$
u(t)=e^{-i t H} u_{-}-i \lambda \int_{-\infty}^{t} e^{-i(t-s) H}\left(|u|^{2 \sigma} u\right)(s) d s=: \Phi(u)(t),
$$

applied in $X_{\gamma_{0}}$ for $\gamma_{0} \ll-1$ and $(p, q, r)$ well chosen. Thanks to Theorem 3.4 and Lemma 4.1 the argument becomes rather close to the proof the second point in Theorem 1.1 for which the reader is referred to, e.g., [9, 14].

Proof of Theorem 1.5. The key step, for the stability of $X_{\gamma_{0}}$ under the action of the map $\Phi$ as well as for the contraction of $\Phi$ on $X_{\gamma_{0}}$ with respect to the weaker norm $\ell_{\gamma \leqslant \gamma_{0}}^{p} L^{q}\left(I_{\gamma} ; L^{r}\right)$ (Kato's method, see [9]: $X_{\gamma_{0}}$ equipped with that norm is complete, so it is not necessary to prove contraction for norms involving derivatives of $\Phi)$, consists in controlling $B\left(|u|^{2 \sigma} u\right)$ in, say, $\ell_{\gamma \leqslant \gamma_{0}}^{p^{\prime}} L^{q^{\prime}}\left(I_{\gamma} ; L^{r^{\prime}}\right)$, for all $B \in\left\{\mathrm{Id}, A_{1}, \ldots, A_{4}\right\}$. This is so thanks to Strichartz estimates, and to the first point in Lemma 4.1 In view of the second point in Lemma 4.1 and Hölder inequality,

$$
\left\|B\left(|u|^{2 \sigma} u\right)\right\|_{\ell_{\gamma \leqslant \gamma_{0}}^{p^{\prime}} L^{q^{\prime}\left(I_{\gamma} ; L^{r^{\prime}}\right)}} \lesssim\|u\|_{\ell_{\gamma \leqslant \gamma_{0}}^{\theta} L^{\omega}\left(I_{\gamma} ; L^{k}\right)}^{2 \sigma}\|B u\|_{\ell_{\gamma \leqslant \gamma_{0}}^{p} L^{q}\left(I_{\gamma} ; L^{r}\right)},
$$

with a constant independent of $\gamma_{0}$, provided that

$$
\frac{1}{p^{\prime}}=\frac{1}{p}+\frac{2 \sigma}{\theta}, \quad \frac{1}{q^{\prime}}=\frac{1}{q}+\frac{2 \sigma}{\omega}, \quad \frac{1}{r^{\prime}}=\frac{1}{r}+\frac{2 \sigma}{k} .
$$

If we can find $(p, q, r)$ with the above algebraic requirements, such that

$$
u \in X_{\gamma_{0}} \Longrightarrow u \in \ell_{\gamma \leqslant \gamma_{0}}^{\theta} L^{\omega}\left(I_{\gamma} ; L^{k}\right),
$$

with $(\theta, \omega, k)$ given like above, for some finite $\theta$, then the result follows, up to taking $\left|\gamma_{0}\right|$ sufficiently large.

Let $u \in X_{\gamma_{0}}$. In view of the last point in Lemma 4.1,

$$
\|u(t)\|_{L^{k}\left(\mathbf{R}^{d}\right)} \leqslant \frac{C}{|t|^{(d-n)(1 / 2-1 / k)}}, \quad t<\pi\left(\gamma_{0}+1\right),
$$

where $C$ depends only on $d, n, k$ and $\left\|u_{-}\right\|_{\Sigma}$. Since $\left|I_{\gamma}\right|=2 \pi$, we infer for any $\omega, \gamma \leqslant \gamma_{0}$,

$$
\|u(t)\|_{L^{\omega}\left(I_{\gamma} ; L^{k}\left(\mathbf{R}^{d}\right)\right)} \leqslant(2 \pi)^{1 / \omega}\|u(t)\|_{L^{\infty}\left(I_{\gamma} ; L^{k}\left(\mathbf{R}^{d}\right)\right)} \lesssim \frac{1}{|\gamma|^{(d-n)(1 / 2-1 / k)}} .
$$

Therefore,

$$
X_{\gamma_{0}} \subset \ell_{\gamma \leqslant \gamma_{0}}^{\theta} L^{\omega}\left(I_{\gamma} ; L^{k}\right) \text { as soon as }(d-n)\left(\frac{1}{2}-\frac{1}{k}\right) \theta>1 \text {. }
$$

We distinguish two cases: $d=2$ and $d \geqslant 3$. 
Case $d=2$. Then necessarily, $n=1$. We take $r=2+\varepsilon$, with $0<\varepsilon \ll 1$. In view of 4.3), we compute:

$$
k=\frac{2 \sigma(2+\varepsilon)}{\varepsilon}, \quad p \frac{4(2+\varepsilon)}{\varepsilon}, \quad q=\frac{2(2+\varepsilon)}{\varepsilon}, \quad \theta=2 \sigma \frac{4+2 \varepsilon}{4+\varepsilon}, \quad \omega=(2+\varepsilon) \sigma,
$$

hence

$$
(d-n)\left(\frac{1}{2}-\frac{1}{k}\right) \theta=\frac{4 \sigma+2(\sigma-1) \varepsilon}{4+\varepsilon} .
$$

This is larger than 1 for $\varepsilon>0$ sufficiently small as soon as $\sigma>1$.

Case $d \geqslant 3$. We consider the largest possible value for $k$, which corresponds to the endpoint of Sobolev embedding,

$$
k=\frac{2 d}{d-2} .
$$

In view of 4.3), we compute

$$
r=\frac{2 d}{d-(d-2) \sigma}
$$

Since the nonlinearity is energy-subcritical, $\sigma<2 /(d-2)$, we have $2 \leqslant r<2 d /(d-2)$, so this value is acceptable. We also have

$$
p=\frac{4 d}{(d-n) \sigma(d-2)}, \quad q=\frac{4}{\sigma(d-2)}, \quad \theta=\frac{4 d \sigma}{2 d-(d-n)(d-2) \sigma},
$$

where the last formula defines a positive $\theta$, since $\sigma<2 /(d-2)$ and $n>0$. We infer

$$
(d-n)\left(\frac{1}{2}-\frac{1}{k}\right) \theta=\frac{d-n}{d} \frac{4 d \sigma}{2 d-(d-n)(d-2) \sigma} .
$$

This quantity is larger than 1 if and only if

$$
\sigma>\frac{2 d}{d+2} \frac{1}{d-n}
$$

which is the condition stated in Theorem 1.5

4.3. Asymptotic completeness for small data. We now turn to the proof of the second part of Theorem 1.5. Resume exactly the same computations as the previous subsection. For $B \in\left\{\operatorname{Id}, A_{1}, \ldots, A_{4}\right\}$,

$$
\|B u\|_{\ell_{\gamma \geqslant 1}^{p} L^{q}\left(I_{\gamma} ; L^{r}\right)} \lesssim\left\|B(0) u_{0}\right\|_{L^{2}}+\|u\|_{\ell_{\gamma \geqslant 1}^{\theta} L^{\omega}\left(I_{\gamma} ; L^{k}\right)}^{2 \sigma}\|B u\|_{\ell_{\gamma \geqslant 1}^{p} L^{q}\left(I_{\gamma} ; L^{r}\right)} .
$$

For $t \geqslant 0$, we estimate, in view of the last point in Lemma4.1

$$
\|u(t)\|_{L^{k}\left(\mathbf{R}^{d}\right)} \leqslant \frac{C}{\langle t\rangle^{(d-n)(1 / 2-1 / k)}}\left\|u_{0}\right\|^{1-\delta}\left(\sum_{j=1}^{4}\left\|A_{j}(t) u\right\|_{L^{2}}\right)^{\delta}
$$

with $\delta=d(1 / 2-1 / k)$. Now let

$$
M(t)=\sum_{j=0}^{4}\left(\left\|A_{j} u\right\|_{\ell_{0 \leqslant \gamma \lesssim t}^{p} L^{q}\left(I_{\gamma} ; L^{r}\right)}+\left\|A_{j} u\right\|_{L^{\infty}\left([0, t] ; L^{2}\right)}\right) .
$$

It satisfies

$$
M(t) \lesssim\left\|u_{0}\right\|_{\Sigma}+M(t)^{1+2 \sigma \delta},
$$

with $\delta$ as above. Recall that standard bootstrap argument: 
Lemma 4.2 (Bootstrap argument). Let $M=M(t)$ be a nonnegative continuous function on $[0, T]$ such that, for every $t \in[0, T]$,

$$
M(t) \leqslant \varepsilon_{1}+\varepsilon_{2} M(t)^{\kappa},
$$

where $\varepsilon_{1}, \varepsilon_{2}>0$ and $\kappa>1$ are constants such that

$$
\varepsilon_{1}<\left(1-\frac{1}{\kappa}\right) \frac{1}{\left(\kappa \varepsilon_{2}\right)^{1 /(\kappa-1)}}, \quad M(0) \leqslant \frac{1}{\left(\kappa \varepsilon_{2}\right)^{1 /(\kappa-1)}} .
$$

Then, for every $t \in[0, T]$, we have

$$
M(t) \leqslant \frac{\kappa}{\kappa-1} \varepsilon_{1} .
$$

We infer that $M$ is bounded for all $t \geqslant 0$ provided that $\left\|u_{0}\right\|_{\Sigma}$ is sufficiently small. Once we know that $A_{j} u \in L^{\infty}\left([0, \infty) ; L^{2}\right)$ for all $j \in\{0, \ldots, 4\}$, we readily infer, by using Strichartz estimates again, that $\left(e^{i t H} A_{j}(t) u\right)_{t \geqslant 0}$ is a Cauchy sequence as $t \rightarrow \infty$, hence the result.

\section{Anisotropic Morawetz EStimates}

In this section, we prove anisotropic Morawetz estimates, as stated in Proposition 1.9

Proof. We proceed as in [16], by working on quadratic quantities which appear naturally in the hydrodynamical reformulation of (1.3). We deduce a monotonicity formula for an appropriate virial quantity and then we infer the a priori bounds for the desired quantities. To shorten the notations, we set $z=(x, y)$. In [16] the calculations rely on the conservation laws for the first two momenta related to the wave function: if $u$ is a solution to (1.3), then we have

$$
\left\{\begin{array}{l}
\partial_{t} \rho+\operatorname{div} J=0 \\
\partial_{t} J+\operatorname{div}(\operatorname{Re}(\nabla \bar{u} \otimes \nabla u))+\lambda \frac{\sigma}{\sigma+1} \nabla \rho^{\sigma+1}+\rho \nabla V=\frac{1}{4} \nabla \Delta \rho,
\end{array}\right.
$$

where $\rho(t, z):=|u(t, z)|^{2}$ and $J(t, z):=\operatorname{Im}(\bar{u} \nabla u)(t, z)$. Let us define the virial potential

$$
I(t):=\frac{1}{2} \iint_{\mathbf{R}^{d} \times \mathbf{R}^{d}} \rho(t, z) a\left(\left|z-z^{\prime}\right|\right) \rho\left(t, z^{\prime}\right) d z d z^{\prime}=\frac{1}{2}\langle\rho, a * \rho\rangle,
$$

where $a$ is a sufficiently smooth weight function which will be chosen later. Here $\langle\cdot, \cdot\rangle$ denotes the scalar product in $L^{2}\left(\mathbf{R}^{d}\right)$. By using (5.1), we see that the time derivative of $I(t)$ reads

$$
\frac{d}{d t} I(t)=-\langle\rho, \nabla a * J\rangle=\iint \rho\left(t, z^{\prime}\right) \nabla a\left(\left|z-z^{\prime}\right|\right) \cdot J(t, z) d z^{\prime} d z=: M(t),
$$

where $M(t)$ is the Morawetz action. By using again the balance laws (5.1) we have

$$
\begin{aligned}
\frac{d}{d t} M(t)= & -\left\langle J, \nabla^{2} a * J\right\rangle+\left\langle\rho, \nabla^{2} a * \operatorname{Re}(\nabla \bar{u} \otimes \nabla u)\right\rangle+\frac{\lambda \sigma}{\sigma+1}\left\langle\rho, \Delta a * \rho^{\sigma+1}\right\rangle \\
& -\langle\rho, \nabla a *(\rho \nabla V)\rangle-\frac{1}{4}\langle\rho, \Delta a * \Delta \rho\rangle \\
= & -\left\langle\operatorname{Im}(\bar{u} \nabla u), \nabla^{2} a * \operatorname{Im}(\bar{u} \nabla u)\right\rangle+\left\langle\rho, \nabla^{2} a *(\nabla \bar{u} \otimes \nabla u)\right\rangle \\
& +\frac{\lambda \sigma}{\sigma+1}\left\langle\rho, \Delta a * \rho^{\sigma+1}\right\rangle-\langle\rho, \nabla a *(\rho \nabla V)\rangle-\frac{1}{4}\langle\rho, \Delta a * \Delta \rho\rangle,
\end{aligned}
$$


where in the second term we dropped the real part because of the symmetry of $\nabla^{2} a$. For the first term we have

$$
\begin{aligned}
\left\langle\operatorname{Im}(\bar{u} \nabla u), \nabla^{2} a * \operatorname{Im}(\bar{u} \nabla u)\right\rangle & =\left\langle\bar{u} \nabla u, \nabla^{2} a *(\bar{u} \nabla u)\right\rangle-\left\langle\operatorname{Re}(\bar{u} \nabla u), \nabla^{2} a * \operatorname{Re}(\bar{u} \nabla u)\right\rangle \\
& =\left\langle\bar{u} \nabla u, \nabla^{2} a *(\bar{u} \nabla u)\right\rangle-\frac{1}{4}\left\langle\nabla \rho, \nabla^{2} a * \nabla \rho\right\rangle,
\end{aligned}
$$

hence we may write

$$
\begin{aligned}
\frac{d}{d t} M(t)= & -\left\langle\bar{u} \nabla u, \nabla^{2} a *(\bar{u} \nabla u)\right\rangle+\left\langle\rho, \nabla^{2} a *(\nabla \bar{u} \otimes \nabla u)\right\rangle \\
& +\frac{1}{4}\left\langle\nabla \rho, \nabla^{2} a * \nabla \rho\right\rangle-\frac{1}{4}\langle\rho, \Delta a * \Delta \rho\rangle \\
& +\frac{\lambda \sigma}{\sigma+1}\left\langle\rho, \Delta a * \rho^{\sigma+1}\right\rangle \\
& -\iint \rho\left(t, z^{\prime}\right) \rho(t, z) \nabla a\left(\left|z-z^{\prime}\right|\right) \cdot \nabla V(z) d z d z^{\prime}
\end{aligned}
$$

Let us consider the second line in (5.3). It is equal to

$$
\frac{1}{4}\left\langle\nabla \rho, \nabla^{2} a * \nabla \rho\right\rangle-\frac{1}{4}\langle\rho, \Delta a * \Delta \rho\rangle=\frac{1}{2}\left\langle\nabla \rho, \nabla^{2} a * \nabla \rho\right\rangle=\frac{1}{2}\langle\nabla \rho, \Delta a * \nabla \rho\rangle .
$$

Now, we notice the first two terms on the right hand side of 5.3 may be rewritten as

$$
\begin{aligned}
&-\left\langle\bar{u} \nabla u, \nabla^{2} a *(\bar{u} \nabla u)\right\rangle+\left\langle\rho, \nabla^{2} a *\right.(\nabla \bar{u} \otimes \nabla u)\rangle= \\
& \frac{1}{2} \iint_{\mathbf{R}^{d} \times \mathbf{R}^{d}} \nabla^{2} a\left(\left|z-z^{\prime}\right|\right) \cdot\left(\bar{u}\left(t, z^{\prime}\right) \nabla \bar{u}(t, z)-\bar{u}(t, z) \nabla \bar{u}\left(t, z^{\prime}\right)\right) \\
& \cdot\left(u\left(t, z^{\prime}\right) \nabla u(t, z)-u(t, z) \nabla u\left(t, z^{\prime}\right)\right) d z^{\prime} d z,
\end{aligned}
$$

which is non-negative if the Hessian matrix $\nabla^{2} a$ is non-negative definite. On the other hand, by choosing the weight $a(|z|)$ depending only on $y \in \mathbf{R}^{d-n}$ the last term in (5.3) involving the potential $V=V(x)$, vanishes. Therefore, the natural choice for the weight is $a(|z|)=|y|$. In this way we have

$$
\frac{d}{d t} M(t) \geqslant \frac{1}{2}\left\langle\nabla_{y} \rho, \Delta_{y} a * \nabla_{y} \rho\right\rangle+\frac{\lambda \sigma}{\sigma+1}\left\langle\rho, \Delta_{y} a * \rho^{\sigma+1}\right\rangle .
$$

Notice that $\Delta_{y} a(|y|)=\frac{d-n-1}{|y|}$ is, up to a multiplicative constant, the integral kernel of the operator $\left(-\Delta_{y}\right)^{-\frac{d-n-1}{2}}$, that is,

$$
\left(\left(-\Delta_{y}\right)^{-\frac{d-n-1}{2}} f\right)(y)=\int_{\mathbf{R}^{d-n}} \frac{c}{\left|y-y^{\prime}\right|} f\left(y^{\prime}\right) d y^{\prime}
$$

Thus, by recalling $z=(x, y)$, we obtain

$$
\begin{aligned}
\iint_{\mathbf{R}^{d} \times \mathbf{R}^{d}} & \frac{1}{\left|y-y^{\prime}\right|} \nabla_{y} \rho\left(t, z^{\prime}\right) \cdot \nabla_{y} \rho(t, z) d z^{\prime} d z \\
& =\iiint_{\mathbf{R}^{n} \times \mathbf{R}^{n} \times \mathbf{R}^{d-n}} \nabla_{y} \rho(t, x, y) \cdot \nabla_{y}\left(-\Delta_{y}\right)^{-\frac{d-n-1}{2}} \rho\left(t, x^{\prime}, y\right) d x d x^{\prime} d y .
\end{aligned}
$$

Hence, if we define the marginal of the mass density

$$
R(t, y):=\int_{\mathbf{R}^{n}} \rho(t, x, y) d x
$$


the last integral also reads

$$
\left.\left.\int_{\mathbf{R}^{d-n}}|| \nabla_{y}\right|^{\frac{3-(d-n)}{2}} R(t, y)\right|^{2} d y .
$$

We now plug this expression into (5.4) and we integrate in time. Furthermore, since $\lambda>0$ the second term in the right hand side in 5.4 is positive. We then infer

$$
\left.\left.\int_{0}^{T} \int_{\mathbf{R}^{d-n}}|| \nabla_{y}\right|^{\frac{3-(d-n)}{2}} R(t, y)\right|^{2} d y d t \leqslant C \sup _{t \in[0, T]} M(t) .
$$

Furthermore, with our choice of the weight $a(|z|)$, we have

$$
M(t)=\iint \rho\left(t, z^{\prime}\right) \frac{y-y^{\prime}}{\left|y-y^{\prime}\right|} \cdot \operatorname{Im}\left(\bar{u} \nabla_{y} u\right)(t, z) d z^{\prime} d z \leqslant\left\|u_{0}\right\|_{L^{2}\left(\mathbf{R}^{d}\right)}^{3}\left\|\nabla_{y} u(t)\right\|_{L^{2}\left(\mathbf{R}^{d}\right)},
$$

where we have used the conservation of the $L^{2}$-norm of $u$ ( $V$ is real-valued). We infer that the right hand side in 5.5 is uniformly bounded,

$$
\left.\left.\int_{0}^{T} \int_{\mathbf{R}^{d-n}}|| \nabla_{y}\right|^{\frac{3-(d-n)}{2}} R(t, y)\right|^{2} d y d t \leqslant C\left\|u_{0}\right\|_{L^{2}\left(\mathbf{R}^{d}\right)}^{3} \sup _{t \in \mathbf{R}}\left\|\nabla_{y} u(t)\right\|_{L^{2}\left(\mathbf{R}^{d}\right)} .
$$

By letting $T$ go to infinity, we prove 1.6.

Remark 5.1. Let us come back to (5.4) in the proof of Proposition 1.9 We see that the second term in the right hand side is nonnegative, and is equal to

$$
\begin{aligned}
& \frac{\lambda \sigma}{\sigma+1} \iint \rho\left(t, z^{\prime}\right) \frac{d-n-1}{\left|y-y^{\prime}\right|} \rho^{\sigma+1}(t, z) d z^{\prime} d z \\
& \qquad \frac{\lambda \sigma}{\sigma+1} \iint \frac{d-n-1}{\left|y-y^{\prime}\right|} \rho^{\frac{\sigma+2}{2}}\left(t, z^{\prime}\right) \rho^{\frac{\sigma+2}{2}}(t, z) d z^{\prime} d z .
\end{aligned}
$$

As before, $\frac{1}{\left|y-y^{\prime}\right|}$ is the integral kernel of $\left(-\Delta_{y}\right)^{-\frac{d-n-1}{2}}$, hence the last integral may be written in the following way,

$$
\left.\left.C \int_{\mathbf{R}^{d-n}}|| \nabla_{y}\right|^{-\frac{d-n-1}{2}} \int_{\mathbf{R}^{n}} \rho^{\frac{\sigma+2}{2}}(t, x, y) d x\right|^{2} d y .
$$

Consequently, from Morawetz estimates we also infer the following a priori bound

$$
\left.\left.\iint_{\mathbf{R} \times \mathbf{R}^{d-n}}|| \nabla_{y}\right|^{-\frac{d-n-1}{2}} \int \rho^{(\sigma+2) / 2}(t, x, y) d x\right|^{2} d y d t \leqslant C\left\|u_{0}\right\|_{L^{2}}^{3} \sup _{t \in \mathbf{R}}\left\|\nabla_{y} u(t)\right\|_{L^{2}} .
$$

This term does not appear in the statement of Proposition 1.9 since (1.6) is the only estimate we are going to use to prove asymptotic completeness.

\section{ASYMPTOTIC COMPLETENESS}

6.1. Outline of the proof and technical remarks. The strategy of the proof of Theorem 1.10 is the same as in e.g. [29] (Section 5.1, for the one-dimensional example), or [1]. Global in time estimates for the solution $u$ are obtained by an inductive bootstrap argument:

Lemma 6.1. Let $t_{0} \in \mathbf{R}, h \in L_{\mathrm{loc}}^{q}\left(\left[t_{0}, \infty\right)\right)$ for some $1 \leqslant q \leqslant \infty$. Suppose that there exist $C_{0}, \eta, \kappa>0$, and $f \in L^{p}\left(\left[t_{0}, \infty\right)\right)$ with $1 \leqslant p<\infty$, such that for all $t^{\prime} \geqslant t \geqslant t_{0}$,

$$
\|h\|_{L^{q}\left(\left[t, t^{\prime}\right]\right)} \leqslant C_{0}+\|f\|_{L^{p}\left(\left[t, t^{\prime}\right]\right)}^{\eta}\|h\|_{L^{q}\left(\left[t, t^{\prime}\right]\right)}^{\kappa} .
$$


Then $h$ is globally integrable,

$$
h \in L^{q}\left(\left[t_{0}, \infty\right)\right) .
$$

Proof. If $\kappa>1$, split $\left[t_{0}, \infty\right)$ into finitely many sub-intervals $I_{j}$, on which $\|f\|_{L^{p}\left(I_{j}\right)}^{\eta} \leqslant \varepsilon_{2}$, with $\varepsilon_{2}=\varepsilon_{2}\left(\eta, f, C_{0}, \kappa\right)$ so small that Lemma4.2 implies

$$
\|h\|_{L^{q}\left(I_{j}\right)} \leqslant \frac{\kappa}{\kappa-1} C_{0}
$$

and the result follows. If $\kappa \leqslant 1$, we may invoke Young inequality $a b \lesssim a^{q}+b^{q^{\prime}}, 1<q<$ $\infty$, to fall back into the first case.

Remark 6.2. When $\kappa>1$, it is crucial in the above lemma that $C_{0}$ is independent of $t, t^{\prime}$. If we suppose for instance that we have inequalities of the form

$$
M\left(t^{\prime}\right) \leqslant M(t)+\|f\|_{L^{p}\left(\left[t, t^{\prime}\right]\right)}^{\eta} M\left(t^{\prime}\right)^{\kappa},
$$

then $M$ need not be bounded. Consider for instance $M(t)=t$ : for $t^{\prime} \geqslant t \geqslant 1$,

$$
t^{\prime}=t+t^{\prime}-t=t+\frac{t^{\prime}-t}{t t^{\prime}} t t^{\prime} \leqslant t+\frac{t^{\prime}-t}{t t^{\prime}}\left(t^{\prime}\right)^{2}
$$

that is,

$$
M\left(t^{\prime}\right) \leqslant M(t)+\left(\int_{t}^{t^{\prime}} \frac{d t}{t^{2}}\right) M\left(t^{\prime}\right)^{2} .
$$

We also need a Gronwall type argument:

Lemma 6.3. Let $t_{0} \in \mathbf{R}, M \in C\left(\left[t_{0}, \infty\right)\right)$. Suppose that there exist $\eta>0$, and $f \in$ $L^{p}\left(\left[t_{0}, \infty\right)\right)$ with $1 \leqslant p<\infty$, such that for all $t^{\prime} \geqslant t \geqslant t_{0}$,

$$
M\left(t^{\prime}\right) \leqslant M(t)+\|f\|_{L^{p}\left(\left[t, t^{\prime}\right]\right)}^{\eta} M\left(t^{\prime}\right) .
$$

Then $M$ is bounded, $M \in L^{\infty}\left(\left[t_{0}, \infty\right)\right)$.

Proof. Split $\left[t_{0}, \infty\right)$ into finitely many sub-intervals $I_{j}$,

$$
\left[t_{0}, \infty\right)=\bigcup_{j=0}^{N} I_{j}, \quad I_{j}=\left[t_{j}, t_{j+1}\right), t_{N+1}=\infty,
$$

on which $\|f\|_{L^{p}\left(I_{j}\right)}^{\eta} \leqslant 1 / 2$. Then

$$
\sup _{t \in I_{j}} M(t) \leqslant 2 M\left(t_{j}\right)
$$

hence

$$
\sup _{t \geqslant t_{0}} M(t) \leqslant 2^{N+1} M\left(t_{0}\right)
$$

Now the goal is to use Strichartz inequalities and Hölder inequality in order to obtain 6.1) with $h(t)=\|u(t)\|_{Z}$ for some suitable $Z$, so that the existence of such a function $f$ follows from the conservation of energy and Morawetz estimates. Lemma 6.3 is then applied to $M(t)=\|B u\|_{L^{p}([0, t] ; Z)}$, where $B \in\left\{A_{1}, \ldots, A_{4}\right\}$. Essentially, the nonlinear estimates of Lemma 6.1 become linear estimates as in Lemma 6.3 because all the vectorfields $A_{j}$ act like first order derivatives on gauge invariant nonlinearities. Note that in view 
of the conservation of energy (see Proposition 1.4) and the identity (4.2), we may replace $h$ at the first step with

$$
h(t)=\sum_{j=0}^{3}\left\|A_{j}(t) u\right\|_{Z},
$$

since the conservations of mass and energy yield an a priori bound in

$$
\Sigma_{\text {anis }}=\left\{f \in L^{2}\left(\mathbf{R}^{d}\right), \quad x f, \nabla_{x} f, \nabla_{y} f \in L^{2}\left(\mathbf{R}^{d}\right)\right\} .
$$

In that case, only the operator $A_{4}$, which contains the large time decay information, is left out. We will follow both approaches below.

At this stage, the strategy may appear relatively standard. The less standard aspect is that the numerology associated to Strichartz estimates in the present framework does not allow to follow directly the above road map by working in $\ell_{\gamma}^{p} L^{q}\left(I_{\gamma}, L^{r}\right)$, with $(p, q, r)$ as in Theorem 3.4. Indeed, apply Hölder inequality to the quantity

$$
\left\||u|^{2 \sigma} u\right\|_{\ell_{\gamma}^{p_{1}^{\prime}} L_{1}^{q_{1}^{\prime}} L_{1}^{r_{1}^{\prime}}},
$$

which appears after the use of inhomogeneous Strichartz estimates, to bound it by

$$
\|u\|_{\ell_{\gamma}^{\theta} L^{\omega} L^{s}}^{(2 \sigma+1)(1-\eta)}\|u\|_{\ell_{\gamma}^{p} L^{q} L^{r}}^{(2 \sigma+1) \eta},
$$

where $\eta \in(0,1)$, and $\left(p_{1}, q_{1}, r_{1}\right),(p, q, r)$ are triplets as in Theorem 3.4 with

$$
\begin{aligned}
& 1=\frac{1}{p_{1}}+\frac{(2 \sigma+1)(1-\eta)}{\theta}+\frac{(2 \sigma+1) \eta}{p}, \\
& 1=\frac{1}{q_{1}}+\frac{(2 \sigma+1)(1-\eta)}{\omega}+\frac{(2 \sigma+1) \eta}{q}, \\
& 1=\frac{1}{r_{1}}+\frac{(2 \sigma+1)(1-\eta)}{s}+\frac{(2 \sigma+1) \eta}{r} .
\end{aligned}
$$

Then since $d-n<d$, we necessarily have $\theta<\omega$, unless $r_{1}=r=2$, a case where Strichartz estimates are not even needed (energy estimate). Unless we proceed as in Section 4, which means unless we use Gagliardo-Nirenberg inequality, we cannot claim that $u \in \ell_{\gamma}^{\theta} L^{\omega} L^{s}$ with $\theta<\omega$, by invoking only Morawetz estimates and the conservation of energy. To overcome this issue, we shall use the Strichartz estimates from Proposition 3.1 (which, in our case, follow essentially from [31], since the harmonic oscillator possesses an eigenbasis): the above space $Z$ will be of the form $L_{y}^{r} L_{x}^{2}$, or a more sophisticated version of it. We prove Theorem 1.10 from the easiest case to the most involved technically: if $d=3$ or 4, a rather straightforward estimate allows to apply Lemmas 6.1 and 6.3, successively. When $d=2$, the same strategy works for $2<\sigma<4$ : since this does not cover the whole range of values for $\sigma$, we directly present another approach, more in the spirit of [32].

6.2. The case $d=4$. In addition to the global existence result stated in Proposition 1.4 Proposition 1.9 yields the information $u \in L_{t}^{4} L_{y}^{4} L_{x}^{2}$. Let $B \in\left\{\mathrm{Id}, A_{1}, A_{2}, A_{3}\right\}$. As recalled above, $B u \in L_{t}^{\infty} L_{x, y}^{2}$ from the conservation of the energy (see also (4.2)). In view of Proposition 3.1 and Hölder inequality, we have, since $\left(\frac{10}{3}, \frac{10}{3}\right)$ is 3 -admissible, for $t \in I$ any interval

$$
\begin{aligned}
\|B u\|_{L_{t}^{10 / 3} L_{y}^{10 / 3} L_{x}^{2}} & \lesssim\left\|u_{0}\right\|_{\Sigma}+\left\||u|^{2 \sigma} B u\right\|_{L_{t}^{10 / 7} L_{y}^{10 / 7} L_{x}^{2}} \\
& \lesssim\left\|u_{0}\right\|_{\Sigma}+\|u\|_{L_{t}^{5 \sigma} L_{y}^{5 \sigma} L_{x}^{\infty}}^{2 \sigma}\|B u\|_{L_{t}^{10 / 3} L_{y}^{10 / 3} L_{x}^{2}} .
\end{aligned}
$$


Since $n=1$, we invoke the standard estimate

to infer

$$
\|f\|_{L^{\infty}(\mathbf{R})} \leqslant \sqrt{2}\|f\|_{L^{2}(\mathbf{R})}^{1 / 2}\left\|\partial_{x} f\right\|_{L^{2}(\mathbf{R})}^{1 / 2},
$$

$$
\|u\|_{L_{t}^{5 \sigma} L_{y}^{5 \sigma} L_{x}^{\infty}} \lesssim\|u\|_{L_{t}^{p} L_{y}^{p} L_{x}^{2}}^{1 / 2}\|u\|_{L_{t}^{10 / 3} L_{y}^{10 / 3} H_{x}^{1}}^{1 / 2}, \quad \text { where } \frac{1}{p}=\frac{4-3 \sigma}{10 \sigma} .
$$

For $\sigma \in\left(\frac{2}{3}, 1\right)$, we have

thus we can always interpolate

$$
\frac{1}{10}<\frac{1}{p}<\frac{3}{10}
$$

$$
\|u\|_{L_{t}^{p} L_{y}^{p} L_{x}^{2}} \leqslant\|u\|_{L_{t}^{4} L_{y}^{4} L_{x}^{2}}^{\alpha}\|u\|_{L_{t}^{q} L_{y}^{q} L_{x}^{2}}^{1-\alpha},
$$

with $0<\alpha<1$, and where $q$ is either 10 or $\frac{10}{3}$. The value 10 is motivated by the fact that the pair $(10,10)$ in 3 -admissible at the level of $\dot{H}^{1}$ (instead of $L^{2}$ so far), and so

$$
\|u\|_{L_{t}^{10} L_{y}^{10} L_{x}^{2}} \lesssim\left\|\nabla_{y} u\right\|_{L_{t}^{10} L_{y}^{30 / 13} L_{x}^{2}}
$$

where the pair $\left(10, \frac{30}{13}\right)$ is 3 -admissible. Letting

$$
h(t)=\sup _{(p, r) 3-\text { admissible }} \sum_{j=0}^{3}\left\|A_{j}(t) u\right\|_{L_{y}^{r} L_{x}^{2}}^{p},
$$

we conclude that this function satisfies, for all interval $I$,

$$
\|h\|_{L^{1}(I)} \lesssim 1+\|u\|_{L^{4}\left(I ; L_{y}^{4} L_{x}^{2}\right)}^{\beta}\|h\|_{L^{1}(I)}^{\kappa},
$$

for some $\beta, \kappa>0$. Lemma 6.1 yields

$$
u, A_{1} u, A_{2} u, A_{3} u \in L^{p}\left(\mathbf{R} ; L_{y}^{r} L_{x}^{2}\right)
$$

for all 3-admissible pairs $(p, r)$. Now taking $B=A_{4}$ in the above estimate, Lemma 6.3 yields $A_{4} u \in L^{p}\left(\mathbf{R} ; L_{y}^{r} L_{x}^{2}\right)$. Theorem 1.10 follows for $d=4$, thanks to the classical arguments recalled in Section 4

6.3. The case $d=3$. We now have the a priori information $\left|\nabla_{y}\right|^{1 / 2}\left(|u|^{2}\right) \in L_{t}^{2} L_{y}^{2} L_{x}^{1}$. In view of Sobolev embedding, we infer

$$
\|u\|_{L_{t}^{4} L_{y}^{8} L_{x}^{2}}^{2}=\left\||u|^{2}\right\|_{L_{t}^{2} L_{y}^{4} L_{x}^{1}} \lesssim\left\|\left|\nabla_{y}\right|^{1 / 2}\left(|u|^{2}\right)\right\|_{L_{t}^{2} L_{y}^{2} L_{x}^{1}}<\infty .
$$

We then proceed as in the case $d=4$. Notice that $(4,4)$ is 2-admissible. For $B \in$ $\left\{\mathrm{Id}, A_{1}, A_{2}, A_{3}\right\}$,

$$
\begin{aligned}
\|B u\|_{L_{t}^{4} L_{y}^{4} L_{x}^{2}} & \lesssim\left\|u_{0}\right\|_{\Sigma}+\left\||u|^{2 \sigma} B u\right\|_{L_{t}^{4 / 3} L_{y}^{4 / 3} L_{x}^{2}} \\
& \lesssim\left\|u_{0}\right\|_{\Sigma}+\|u\|_{L_{t}^{4 \sigma} L_{y}^{4 \sigma} L_{x}^{\infty}}^{2 \sigma}\|B u\|_{L_{t}^{4} L_{y}^{4} L_{x}^{2}}
\end{aligned}
$$

We interpolate the nonlinear potential by

$$
\|u\|_{L_{t}^{4 \sigma} L_{y}^{4 \sigma} L_{x}^{\infty}} \lesssim\|u\|_{L_{t}^{\frac{8 \sigma}{2-\sigma}} \frac{8 \sigma}{L_{y}^{2-\sigma}} L_{x}^{2}}^{\theta}\|u\|_{L_{t}^{4} L_{y}^{4} H_{x}^{1}}^{1-\theta}, \quad \theta=\frac{2(\sigma-1)}{3 \sigma-2},
$$

and we note that $0<\theta<1 / 2$ since $1<\sigma<2$. We interpolate again to introduce the quantity controlled by Morawetz estimate,

$$
\|u\|_{L_{t}^{\frac{8 \sigma}{2-\sigma}} L_{y}^{\frac{8 \sigma}{2-\sigma}} L_{x}^{2}} \leqslant\|u\|_{L_{t}^{\infty} L_{y}^{\frac{8(3 \sigma-2)}{2-\sigma}} L_{x}^{2}}^{1-\alpha}\|u\|_{L_{t}^{4} L_{y}^{8} L_{x}^{2}}^{\alpha}, \quad \alpha=\frac{2-\sigma}{2 \sigma} \in(0,1) .
$$


Notice that $y \in \mathbf{R}^{2}$, so by Sobolev embedding,

$$
\|u\|_{L_{t}^{\infty} L_{y} \frac{8(3 \sigma-2)}{2-\sigma} L_{x}^{2}} \lesssim\|u\|_{L_{t}^{\infty} H_{y}^{1} L_{x}^{2}} \lesssim\left\|u_{0}\right\|_{\Sigma}
$$

where the last inequality stems from Proposition 1.4 We can then conclude as in the case $d=4$, by considering first

$$
h(t)=\sum_{j=0}^{3}\left\|A_{j}(t) u\right\|_{L_{y}^{4} L_{x}^{2}}^{4},
$$

hence Theorem 1.10 in the case $d=3$.

6.4. The case $d=2$. In this case, instead of working mainly with an $L^{2}$ regularity in $x$, we consider a Banach algebra. Since $x$ is associated to a harmonic oscillator, it is not sensible to require only a Sobolev type regularity of the form $H_{x}^{s}$, since the harmonic oscillator rotates the phase space (see e.g. [6]). Instead, we consider the domain of the fractional harmonic oscillator: for $s \geqslant 0$ a real number, let

$$
\Sigma_{x}^{s}=\left\{f \in L^{2}(\mathbf{R}), \quad\|f\|_{\Sigma_{x}^{s}}:=\|f\|_{L^{2}(\mathbf{R})}+\left\|\left(-\partial_{x}^{2}+x^{2}\right)^{s / 2} f\right\|_{L^{2}(\mathbf{R})}<\infty\right\} .
$$

We know from [3, Theorem 2.1] that (for any nonnegative real number $s$ ) the above norm enjoys the following equivalence,

$$
\|f\|_{\Sigma_{x}^{s}} \sim\|f\|_{L^{2}(\mathbf{R})}+\|f\|_{\dot{H}^{s}(\mathbf{R})}+\left\||x|^{s} f\right\|_{L^{2}(\mathbf{R})} .
$$

In particular, $\Sigma_{x}^{s}$ is a Banach algebra as soon as $s>1 / 2$.

Lemma 6.4. Let $d \geqslant 2,1 \leqslant n \leqslant d-1$, and $0 \leqslant s \leqslant 1$. Then for all $u_{0} \in L_{y}^{2}\left(\mathbf{R}^{d-n} ; \Sigma_{x}^{s}\right)$, all $(d-n)$-admissible pairs $\left(p_{1}, r_{1}\right)$ and $\left(p_{2}, r_{2}\right)$, there exists $C_{r_{1}, r_{2}}$ such that for all interval $I \ni 0$, the solution to

$$
i \partial_{t} u=H u+F, \quad u_{\mid t=0}=u_{0},
$$

satisfies:

$$
\|u\|_{L_{t}^{p_{1}}\left(I ; L_{y}^{r_{1}} \Sigma_{x}^{s}\right)} \leqslant C_{r_{1}, r_{2}}\left(\left\|u_{0}\right\|_{L_{y}^{2} \Sigma_{x}^{s}}+\|F\|_{L_{t}^{p_{2}^{\prime}\left(I ; L_{y}^{r_{2} \Sigma_{x}^{s}}\right)}}\right) .
$$

Proof. For $s=0$, the result is a particular case of Proposition 2.2 (or Proposition 3.1). For $s=1$, apply the vector-fields $A_{1}$ and $A_{2}$ to obtain the result from the case $s=0$ and the identity (4.2). The case $0<s<1$ follows by interpolation.

Morawetz estimate yields $|u|^{2} \in L_{t}^{2} \dot{H}_{y}^{1} L_{x}^{1}$. In view of the one-dimensional inequality

$$
\|f\|_{L_{y}^{\infty}} \lesssim\|f\|_{L_{y}^{1}}^{1 / 3}\left\|\partial_{y} f\right\|_{L_{y}^{2}}^{2 / 3}
$$

we infer the analogue of the property established in [29], $u \in L_{t}^{6} L_{y}^{\infty} L_{x}^{2}$.

On the other hand, the conservation of mass and energy yields

$$
u \in L_{t}^{\infty} H_{y}^{1 / 2-\alpha} \Sigma_{x}^{1 / 2+\alpha}, \quad 0 \leqslant \alpha \leqslant \frac{1}{2} .
$$

Sobolev embedding yields $(d-n=1)$

$$
u \in L_{t}^{\infty} L_{y}^{1 / \alpha} \Sigma_{x}^{1 / 2+\alpha}, \quad 0 \leqslant \alpha \leqslant \frac{1}{2} .
$$

By interpolation, we infer

$$
u \in L_{t}^{6 / \alpha} L_{y}^{1 /(\alpha(1-\alpha))} \Sigma_{x}^{1 / 2+\delta}, \quad 0<\alpha<\frac{1}{2}, \quad \delta=(1-\alpha)\left(\frac{1}{2}+\alpha\right)-\frac{1}{2}>0 .
$$


For $\alpha \in(0,1 / 2)$ to be fixed, and $(p, r)$ a 1-admissible pair to fix too, write

$$
\left\||u|^{2 \sigma} u\right\|_{L_{t}^{p^{\prime}} L_{y}^{r^{\prime}} \mathcal{H}_{x}} \lesssim\|u\|_{L_{t}^{p} L_{y}^{r} \mathcal{H}_{x}}^{(2 \sigma+1) \eta}\|u\|_{L_{t}^{6 / \alpha} L_{y}^{1 /(\alpha(1-\alpha))} \mathcal{H}_{x}}^{(2 \sigma+1)(1-\eta)},
$$

where $\mathcal{H}_{x}=\Sigma_{x}^{1 / 2+\delta}$, and $\eta \in(0,1)$. We have used Hölder inequality, with

$$
\begin{aligned}
& 1=\frac{(2 \sigma+1) \eta+1}{p}+(2 \sigma+1)(1-\eta) \frac{\alpha}{6} \\
& 1=\frac{(2 \sigma+1) \eta+1}{r}+(2 \sigma+1)(1-\eta) \alpha(1-\alpha) .
\end{aligned}
$$

Recalling that $(p, r)$ is 1-admissible, we infer that necessarily,

$$
\frac{5}{4}=\frac{(2 \sigma+1) \eta}{4}+(2 \sigma+1)(1-\eta) \alpha\left(\frac{2}{3}-\frac{\alpha}{2}\right) .
$$

Now let $0<\varepsilon \ll 1$, and define $\eta$ by the identity

$$
5=(2 \sigma+1) \eta+\varepsilon
$$

Since $\sigma>2$, for $\varepsilon$ sufficiently small, this defines indeed $\eta \in(0,1)$. Then, up to decreasing $\varepsilon$, fix $0<\alpha<1 / 2$ so that

$$
(2 \sigma+1)(1-\eta) \alpha\left(\frac{2}{3}-\frac{\alpha}{2}\right)=\varepsilon .
$$

To define all the parameters, we now recall that $r$ is given by 6.3): we check that $2<r<$ 6 , with $r \approx 6$. Once all these parameters are fixed, Strichartz inequality yields

$$
\|u\|_{L_{t}^{p} L_{y}^{r} \mathcal{H}_{x}} \lesssim\left\|u_{0}\right\|_{\Sigma}+\|u\|_{L_{t}^{6 / \alpha} L_{y}^{1 /(\alpha(1-\alpha))} \mathcal{H}_{x}}^{2 \sigma-4+\varepsilon}\|u\|_{L_{t}^{p} L_{y}^{r} \mathcal{H}_{x}}^{5-\varepsilon} .
$$

Following the same argument as in other dimensions, we infer $u \in L^{p}\left(\mathbf{R} ; L_{y}^{r} \mathcal{H}_{x}\right)$, and thus $u \in L^{p_{1}}\left(\mathbf{R} ; L_{y}^{r_{1}} \mathcal{H}_{x}\right)$ for all 1-admissible pairs $\left(p_{1}, r_{1}\right)$.

For $B \in\left\{A_{0}, \ldots, A_{4}\right\}$, we also have, on any interval $I=\left[t_{0}, t_{1}\right]$,

$$
\begin{aligned}
\|B u\|_{L_{t}^{p} L_{y}^{r} L_{x}^{2}} & \lesssim\left\|B\left(t_{0}\right) u\right\|_{L^{2}}+\|u\|_{L_{t}^{6 / \alpha} L_{y}^{1 /(\alpha(1-\alpha))} L_{x}^{\infty}}^{2 \sigma-4+\varepsilon}\|u\|_{L_{t}^{p} L_{y}^{r} L_{x}^{\infty}}^{4-\varepsilon}\|B u\|_{L_{t}^{p} L_{y}^{r} L_{x}^{2}} \\
& \lesssim\left\|B\left(t_{0}\right) u\right\|_{L^{2}}+\|u\|_{L_{t}^{6 / \alpha} L_{y}^{1 /(\alpha(1-\alpha))} \mathcal{H}_{x}}^{2 \sigma-4+\varepsilon}\|u\|_{L_{t}^{p} L_{y}^{r} \mathcal{H}_{x}}^{4-\varepsilon}\|B u\|_{L_{t}^{p} L_{y}^{r} L_{x}^{2}},
\end{aligned}
$$

where we have used the embedding $\mathcal{H}_{x} \subset L_{x}^{\infty}$. Invoking Lemma 6.3, we conclude $B u \in L^{p}\left(\mathbf{R} ; L_{y}^{r} L_{x}^{2}\right)$, hence $B u \in L^{p_{1}}\left(\mathbf{R} ; L_{y}^{r_{1}} L_{x}^{2}\right)$ for all 1-admissible pairs $\left(p_{1}, r_{1}\right)$. Theorem 1.10 then follows in the case $d=2$.

\section{APPENDiX A. EXISTENCE OF LONG RANGE EFFECTS: FORMAL PROOF}

Lemma A.1. Let $f \in L^{2}\left(\mathbf{R}^{n}\right)$ and $g \in L^{2}\left(\mathbf{R}^{d-n}\right)$. Consider the solution $u$ to

$$
i \partial_{t} u=H u, \quad u(0, x, y)=f(x) g(y) .
$$

Then as $t \rightarrow+\infty$,

$$
u(t, x, y)=e^{i t H_{1}} f(x) \otimes \frac{1}{(i t)^{(d-n) / 2}} \mathcal{F}_{2} g\left(\frac{y}{t}\right) e^{i \frac{|y|^{2}}{2 t}}+o(1) \text { in } L^{2}\left(\mathbf{R}^{d}\right),
$$

where $\mathcal{F}_{2}$ stands for the partial Fourier transform with respect to $y$. 
The lemma is straightforward, since

$$
u(t, x, y)=e^{i t H_{1}} f(x) \otimes e^{i t H_{2}} g(y) .
$$

Following the formal argument given in [14], the above lemma suggests that for $\sigma \leqslant$ $1 /(d-n)$, long range effects are present in (1.3). Assume $\lambda=1$ for simplicity. Let $u \in C\left([T, \infty) ; L^{2}\left(\mathbf{R}^{d}\right)\right)$ be a solution of (1.3) such that there exists $u_{+} \in L^{2}\left(\mathbf{R}^{d}\right)$ with

$$
u_{+}(x, y)=f(x) g(y) \text { and }\left\|u(t)-e^{-i t H} u_{+}\right\|_{L^{2}} \underset{t \rightarrow+\infty}{\longrightarrow} 0 .
$$

Formal computations indicate that necessarily, $u_{+} \equiv 0$ and $u \equiv 0$ : the linear and nonlinear dynamics are no longer comparable, due to long range effects. To see this, let $\psi=\psi_{1} \otimes \psi_{2}$ with $\psi_{1} \in C_{0}^{\infty}\left(\mathbf{R}^{n}\right), \psi_{2} \in C_{0}^{\infty}\left(\mathbf{R}^{d-n}\right)$, and $t_{2} \geqslant t_{1} \geqslant T$. By assumption,

$$
\left\langle\psi, e^{i t_{2} H} u\left(t_{2}\right)-e^{i t_{1} H} u\left(t_{1}\right)\right\rangle=-i \int_{t_{1}}^{t_{2}}\left\langle e^{-i t H} \psi,\left(|u|^{2 \sigma} u\right)(t)\right\rangle d t
$$

goes to zero as $t_{1}, t_{2} \rightarrow+\infty$. The above lemma implies that for $t \rightarrow+\infty$, we have

$$
\left\langle e^{-i t H} \psi,\left(|u|^{2 \sigma} u\right)(t)\right\rangle \approx \frac{1}{t^{(d-n)(\sigma+1)}} \int_{\mathbf{R}^{n}} F(t, x) d x \int_{\mathbf{R}^{d-n}} G\left(\frac{y}{t}\right) d y,
$$

for $F=e^{-i t H_{1}} \psi_{1}\left|e^{-i t H_{1}} f\right|^{2 \sigma} \overline{e^{-i t H_{1} f}}$ and $G=\mathcal{F}_{2} \psi_{2}\left|\mathcal{F}_{2} g\right|^{2 \sigma} \overline{\mathcal{F}_{2} g}$. With the change of variable $y \mapsto t y$, the above integral is equal to

$$
\frac{1}{t^{(d-n) \sigma}}\left(\int_{\mathbf{R}^{n}} F(t, x) d x\right)\left(\int_{\mathbf{R}^{d-n}} G(y) d y\right) .
$$

Now $F$ is periodic in time, with arbitrary mean value. But $t \mapsto 1 / t^{(d-n) \sigma}$ is not integrable, so the above quantity is not integrable in time, unless $u_{+}=0$. The conservation of mass then implies $u \equiv 0$.

Acknowledgments. P. Antonelli and J. Drumond Silva would like to thank the kind hospitality of the Institute of Mathematics at the Université Montpellier 2, where part of this work was developed. The authors are grateful to Nicola Visciglia for fruitful discussions.

\section{REFERENCES}

[1] V. BAniCA, R. CARles, And T. DuYCKAERTS, On scattering for NLS: from Euclidean to hyperbolic space, Discrete Contin. Dyn. Syst., 24 (2009), pp. 1113-1127.

[2] J. E. BARAB, Nonexistence of asymptotically free solutions for nonlinear Schrödinger equation, J. Math. Phys., 25 (1984), pp. 3270-3273.

[3] N. Ben Abdallah, F. CAstella, And F. MÉHAts, Time averaging for the strongly confined nonlinear Schrödinger equation, using almost periodicity, J. Differential Equations, 245 (2008), pp. 154-200.

[4] J. Bourgain, Fourier restriction phenomena for certain lattice subsets and applications to nonlinear evolution equations I, Schrödinger equations, Geom. Funct. Anal., 3 (1993), pp. 107-156.

[5] R. CARLES, Linear vs. nonlinear effects for nonlinear Schrödinger equations with potential, Commun. Contemp. Math., 7 (2005), pp. 483-508.

[6] - On the Cauchy problem in Sobolev spaces for nonlinear Schrödinger equations with potential, Portugal. Math. (N. S.), 65 (2008), pp. 191-209.

[7] - Nonlinear Schrödinger equation with time dependent potential, Commun. Math. Sci., 9 (2011), pp. 937-964.

[8] R. CARles AND L. Miller, Semiclassical nonlinear Schrödinger equations with potential and focusing initial data, Osaka J. Math., 41 (2004), pp. 693-725.

[9] T. CAZENAVE, Semilinear Schrödinger equations, vol. 10 of Courant Lecture Notes in Mathematics, New York University Courant Institute of Mathematical Sciences, New York, 2003.

[10] S. CuCCAGNA AND N. Visciglia, On asymptotic stability of ground states of NLS with a finite bands periodic potential in 1D, Trans. Amer. Math. Soc., 363 (2011), pp. 2357-2391.

[11] J. DEREZIŃSKI AND C. GÉRARD, Scattering theory of quantum and classical N-particle systems, Texts and Monographs in Physics, Springer Verlag, Berlin Heidelberg, 1997. 
[12] D. Fujiwara, Remarks on the convergence of the Feynman path integrals, Duke Math. J., 47 (1980), pp. 559-600.

[13] J. GinibRE, Le problème de Cauchy pour des EDP semi-linéaires périodiques en variable d'espace, Astérisque, (1995), pp. Exp. No. 796, p. 163-187. Séminaire Bourbaki, Vol. 1994/95.

[14] J. Ginibre, An introduction to nonlinear Schrödinger equations, in Nonlinear waves (Sapporo, 1995), R. Agemi, Y. Giga, and T. Ozawa, eds., GAKUTO International Series, Math. Sciences and Appl., Gakkōtosho, Tokyo, 1997, pp. 85-133.

[15] J. Ginibre And G. Velo, On a class of nonlinear Schrödinger equations. II Scattering theory, general case, J. Funct. Anal., 32 (1979), pp. 33-71.

[16] J. Ginibre And G. Velo, Quadratic Morawetz inequalities and asymptotic completeness in the energy space for nonlinear Schrödinger and Hartree equations, Quart. Appl. Math., 68 (2010), pp. 113-134.

[17] B. Grébert And L. Thomann, KAM for the quantum harmonic oscillator, Comm. Math. Phys., 307 (2011), pp. 383-427.

[18] Z. HANI AND B. PAUS ADER, On scattering for the quintic defocusing nonlinear Schrödinger equation on $\mathbb{R} \times \mathbb{T}^{2}$, Comm. Pure Appl. Math., (2013). Archived as http://arxiv.org/abs/1205.6136

[19] C. Josserand And Y. Pomeau, Nonlinear aspects of the theory of Bose-Einstein condensates, Nonlinearity, 14 (2001), pp. R25-R62.

[20] L. KapitANS Ki, I. RodNiAns Ki, AND K. YAJIMA, On the fundamental solution of a perturbed harmonic oscillator, Topol. Methods Nonlinear Anal., 9 (1997), pp. 77-106.

[21] M. KeEl AND T. TAO, Endpoint Strichartz estimates, Amer. J. Math., 120 (1998), pp. 955-980.

[22] S. B. KUKSIN, Nearly integrable infinite-dimensional Hamiltonian systems, vol. 1556 of Lecture Notes in Mathematics, Springer-Verlag, Berlin, 1993.

[23] L. D. LANDAU AND E. M. LifshitZ, Quantum mechanics: non-relativistic theory. Course of Theoretical Physics, Vol. 3, Addison-Wesley Series in Advanced Physics, Pergamon Press Ltd., London-Paris, 1958. Translated from the Russian by J. B. Sykes and J. S. Bell.

[24] H. P. McKeAn AND E. TRUBowitz, The spectral class of the quantum-mechanical harmonic oscillator, Comm. Math. Phys., 82 (1981/82), pp. 471-495.

[25] F. G. MEHLER, Ueber die Entwicklung einer Function von beliebig vielen Variablen nach Laplaceseschen Functionen höherer Ordnung, J. reine und angew. Math., 66 (1866), pp. 161-176.

[26] Y.-G. ОH, Cauchy problem and Ehrenfest's law of nonlinear Schrödinger equations with potentials, J. Diff. Eq., 81 (1989), pp. 255-274.

[27] T. OZAWA, Long range scattering for nonlinear Schrödinger equations in one space dimension, Comm. Math. Phys., 139 (1991), pp. 479-493.

[28] L. Pitaevs Kil And S. Stringari, Bose-Einstein condensation, vol. 116 of International Series of Monographs on Physics, The Clarendon Press Oxford University Press, Oxford, 2003.

[29] F. Planchon and L. Vega, Bilinear virial identities and applications, Ann. Sci. Éc. Norm. Supér. (4), 42 (2009), pp. 261-290.

[30] M. ReEd AND B. Simon, Methods of modern mathematical physics. II. Fourier analysis, self-adjointness, Academic Press [Harcourt Brace Jovanovich Publishers], New York, 1975.

[31] N. Tzvetkov AND N. Visciglia, Small data scattering for the nonlinear Schrödinger equation on product spaces, Comm. Partial Differential Equations, 37 (2012), pp. 125-135.

[32] N. ViscigLiA. Private communication on a joint work in progress, 2013.

[33] D. YAfAEV, Scattering theory: some old and new problems, vol. 1735 of Lecture Notes in Mathematics, Springer-Verlag, Berlin, 2000.

[34] S. ZELDITCH, Reconstruction of singularities for solutions of Schrödinger's equation, Comm. Math. Phys., 90 (1983), pp. 1-26.

Centro di Ricerca Matematica Ennio De Giorgi, Piazza dei CaValieri, 3, 56100 Pis a, italy

E-mail address: paolo.antonelli@sns.it

CNRS \& Univ. Montpellier 2, Mathématiques, CC 051, 34095 Montpellier, France

E-mail address: Remi.Carles@math.cnrs.fr

Center for Mathematical Analysis, Geometry and Dynamical Systems, Departamento De Matemática, Instituto Superior Técnico, Universidade de Lisboa, Av. Rovisco Pais, 1049001 Lisboa, Portugal

E-mail address: jsilva@math.ist.utl.pt 